\title{
Development
}

\section{Tracking Mitochondrial Density and Positioning along a Growing Neuronal Process in Individual $C$. elegans Neuron Using a Long-Term Growth and Imaging Microfluidic Device}

\author{
Sudip Mondal, ${ }^{1,2}$ Jyoti Dubey, ${ }^{1,3,4,7}$ Anjali Awasthi,, ${ }^{1,5}$ Guruprasad Reddy Sure, ${ }^{1,6}$ (D)Amruta Vasudevan, ${ }^{3}$ \\ and Sandhya P. Koushika ${ }^{3}$
}

https://doi.org/10.1523/ENEURO.0360-20.2021

${ }^{1}$ National Centre for Biological Sciences, Tata Institute of Fundamental Research, Bangalore, Karnataka 560065, India, ${ }^{2}$ Department of Mechanical Engineering, The University of Texas at Austin, Austin, Texas 78712, ${ }^{3}$ Department of Biological Sciences, Tata Institute of Fundamental Research, Mumbai, Maharashtra 400005, India, ${ }^{4}$ Institute for Stem Cell Science and Regenerative Medicine, Bangalore, Karnataka 560065, India, ${ }^{5}$ Department of Biological Sciences, Birla Institute of Technology and Science, Pilani, Rajasthan 333031, India, ${ }^{6}$ Sastra University, Thirumalaisamudram, Tamil Nadu 613401, India, and ${ }^{7}$ Manipal Academy of Higher Education, Manipal, Karnataka 576104, India

\section{Visual Abstract}

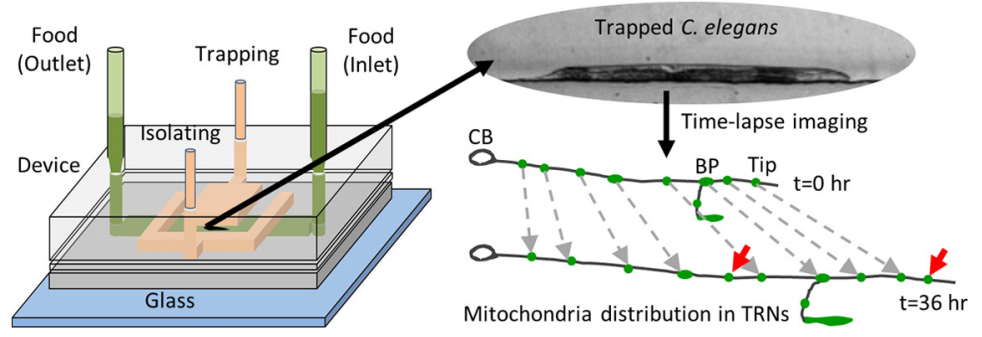

The long cellular architecture of neurons requires regulation in part through transport and anchoring events to distribute intracellular organelles. During development, cellular and subcellular events such as organelle additions and their recruitment at specific sites on the growing axons occur over different time scales and often show interanimal variability thus making it difficult to identify specific phenomena in population averages. To measure the variability in subcellular events such as organelle positions, we developed a microfluidic device to feed and immobilize Caenorhabditis elegans for high-resolution imaging over several days. The microfluidic device enabled long-term imaging of individual animals and allowed us to investigate organelle density using

\section{Significance Statement}

Axonal transport of mitochondria is required for the normal function and health of a developing animal with continuously growing axonal processes. Existing technologies are unable to monitor the addition of a new mitochondrion in a growing axon in vivo, as it requires continuous or intermittent tracking of the same individual neuron over several hours to days. We have developed a microfluidic device that enables long-term high-resolution imaging of individual Caenorhabditis elegans in an anesthetic-free setting. Using this device, we observe that the addition of a new mitochondrion can occur anywhere along the entire neuronal process, likely mediated by actively transported mitochondria, and at docking sites that occur with high probability when the separation between adjacent mitochondria crosses $24 \mu \mathrm{m}$ threshold. 
mitochondria as a testbed in a growing neuronal process in vivo. Subcellular imaging of an individual neuron in multiple animals, over $36 \mathrm{~h}$ in our microfluidic device, shows the addition of new mitochondria along the neuronal process and an increase in the accumulation of synaptic vesicles (SVs) at synapses. Long-term imaging of individual $C$. elegans touch receptor neurons (TRNs) shows that the addition of new mitochondria takes place along the entire neuronal process length at a rate of $\sim 0.6$ mitochondria/h. The threshold for the addition of a new mitochondrion occurs when the average separation between the two preexisting mitochondria exceeds $24 \mu \mathrm{m}$. Our assay provides a new opportunity to move beyond simple observations obtained from in vitro assays to allow the discovery of genes that regulate positioning of mitochondria in neurons.

Key words: C. elegans; touch receptor neurons; mitochondria; intermitochondrial distances; development; microfluidic device; long-term imaging

\section{Introduction}

Development and maturation of neurons occur through the processes of cell birth, cell differentiation, cell migration, neuronal process outgrowth, dendritic arborization, synaptic growth, and organelle transport (Hobert, 2010; Smith et al., 2010; Varier and Kaiser, 2011; Smith and Gallo, 2018). Complex cellular architectures present in polarized cells such as neurons need the transport of organelles to support many energy-dependent processes such as neurotransmission (Sheng and Cai, 2012). One such organelle is mitochondria that are essential for neuronal function and thought to contribute to various disease pathologies (Liu et al., 2012; Millecamps and Julien, 2013). Only $\sim 20 \%$ of a large number of mitochondria in a neuronal process move and their halting depend on molecular motors, calcium, and other signaling molecules (Kang et al., 2008; Chen and Sheng, 2013; Schwarz, 2013; Sun et al., 2013; Misgeld and Schwarz, 2017).

Received August 16, 2020; accepted May 6, 2021; First published May 21, 2021.

S.M. and S.P.K. are authors of a pending patent on the microfluidic growth and imaging device (patent application 640/CHE/2011). All other authors declare no competing financial interests.

Author contributions: S.M. and S.P.K. designed research; S.M., J.D., A.A., G.R.S., and A.V. performed research; S.M. and J.D. analyzed data; S.M. and S. P.K. wrote the paper.

This work was supported by research funding from Department of Biotechnology (S.P.K. and S.M.), Department of Science and Technology (S. M.), Department of Atomic Energy (1303/2/2019/R\&D-II/DAE/2079), and PhD fellowship from Council of Scientific and Industrial Research (G.R.S.) and Council of Scientific and Industrial Research - University Grants Commission (J.D.). The spinning disc was supported by Department of Atomic Energy PRISM 12-R\&D-IMS-5.02.0202 (to S.P.K.), and the Howard Hughes Medical Institute International Early Career Scientist Grant 55007425 (to S.P.K.). Confocal microscopes were supported by the Department of Science and Technology-Centre for Nanotechnology Grant SR/55/NM-36-2005.

Acknowledgements: We thank Anyaa Mittal and Arpan Agnihotri for their help with microfluidic device fabrication and growing $C$. elegans inside microfluidic devices; Gautam Menon for useful discussion on mitochondria transport imaging and image analysis; Sangeetha lyer (Perlara Labs) and Souvik Modi for suggestions on our manuscript; and the reviewers for their thoughtful comments. We also thank the Central Imaging and Flow Cytometry imaging facility, National Centre for Biological Sciences for use of the confocal microscopes. S.P.K. made js/s609 in Mike Nonet's Laboratory.

Correspondence should be addressed to Sudip Mondal at sudipmondal1979@ gmail.com or Sandhya P. Koushika at spkoushika@tifr.res.in.

https://doi.org/10.1523/ENEURO.0360-20.2021 Copyright $@ 2021$ Mondal et al.

This is an open-access article distributed under the terms of the Creative Commons Attribution 4.0 International license, which permits unrestricted use, distribution and reproduction in any medium provided that the original work is properly attributed.
Mitochondrial trafficking continues throughout development when neurons undergo elongation over several fold and offers a unique opportunity to demonstrate the utility of long-term imaging in individual neurons. Time-lapse imaging of rapidly transported mitochondria in neuronal cultures over a few hours has identified that moving mitochondria preferentially dock between pairs of preexisting docked mitochondria (Miller and Sheetz, 2004) and thus help maintain a uniform mitochondrial distribution along axons (Miller and Sheetz, 2004; Misko et al., 2012). Thus far, many invertebrate axons have not typically been reported to have general anatomic features such as nodes of Ranvier enriched in mitochondria as observed in vertebrate neurons (Chiu, 2011; Ohno et al., 2011). Multiple neurons, both in vivo and in non-myelinated neurons in culture, show that the density of mitochondria is invariant (Wang and Schwarz, 2009a; Morsci et al., 2016; Misgeld and Schwarz, 2017; Sure et al., 2018). The majority of mitochondria in Caenorhabditis elegans touch receptor neurons (TRNs) have been reported to be present at actin-rich regions (Sood et al., 2018) and the density of mitochondria in adult animals remains largely invariant (Morsci et al., 2016; Sure et al., 2018). Despite averages being tightly regulated, interanimal variability is shown to exist even in nearly invariant cell lineages (Keil et al., 2017). Developing and aging neurons show variability in mitochondrial density and axonal length in a clonal genetic population (O'Toole et al., 2008), show heterogeneity of mitochondrial dynamics within individual cells (Sison et al., 2017), and show differential mitochondrial dynamics and distribution within different neuron segments (Obashi and Okabe, 2013). To investigate where mitochondria are added in vivo, one needs to analyze each mitochondrion along the entire neuronal process length, which can elongate significantly, i.e., $>5$-fold over $\sim 3 \mathrm{~d}$ in the same $C$. elegans animal.

Developmental events can be easily tracked in C. elegans using a microfluidic platform. Several microfluidic platforms have been developed for $C$. elegans to carry out high-resolution imaging (Chalasani et al., 2007; Mondal et al., 2011, 2012; Cáceres et al., 2012), high-throughput screening (Chung et al., 2008; Mondal et al., 2016, 2018), long-term imaging (Hulme et al., 2010; Krajniak and Lu, 2010; Xian et al., 2013; Keil et al., 2017; Atakan et al., 2019), and neuronal axotomy (Guo et al., 2008; Samara et al., 2010). Microfluidic device immobilization avoids alterations in $C$. elegans physiological processes caused by anesthetic immobilization (Guo et al., 2008; Mondal et al., 
2011, 2012; Mondal and Koushika, 2014). Anesthetic-free immobilization can prevent mechanical stress induced by chemical immobilization methods that change muscle tone and body stiffness, leading to artificial elongation or contraction of the overall body length (Petzold et al., 2011). C. elegans can also be cultured by feeding them with bacterial cultures inside microfluidic devices (Krajniak and Lu, 2010; Krajniak et al., 2013; Xian et al., 2013; Lee et al., 2014; Gokce et al., 2017; Atakan et al., 2019). Microfluidic devices allow time-lapse neuronal imaging in well-fed animals for up to $24 \mathrm{~h}$ (Lee et al., 2014). In aging studies, long-term microfluidic devices have been used to grow $C$. elegans and acquire low-resolution images to quantify brood size and monitor locomotion (Xian et al., 2013; Rahman et al., 2018; Atakan et al., 2019). Recently, neuronal branching dynamics and lineage patterns were monitored using an imaging platform from an animal growing over $3 d$ (Keil et al., 2017). As yet, there is no study tracking subcellular events long-term such as monitoring organelle distributions or accumulations over time. To track cellular and subcellular events in identified individual animals, we developed a polydimethylsiloxane (PDMS) microfluidic device to grow $C$. elegans, completely immobilize animals in the same orientation, and image the same individual neuronal process throughout development.

Here, we describe the design and optimization of our microfluidic device and demonstrate its utility in capturing high-resolution fluorescence images of the same posterior lateral TRNs across $36 \mathrm{~h}$ of $C$. elegans development. Mitochondrial distribution along the neuronal process changes over time, however, the density of mitochondria was similar across different developmental stages. Our data suggest that maintenance of density likely occurs through the addition of a new mitochondrion between two mitochondria that are separated by at least $24 \mu \mathrm{m}$. Our device can also be used to study other events such as the accumulation of synaptic vesicles (SVs) at the synapses, occurring over several days.

\section{Materials and Methods}

\section{Growth and imaging device fabrication}

Our microfluidic device was fabricated using soft lithography of PDMS from SU8 resist features on silicon substrates (Whitesides et al., 2001). The device design consists of two photomasks designed in Clewin software and printed using a high-resolution laser plotter (Fine Line Imaging). The flow layer is comprised of a single $10-\mathrm{mm}-$ long and $300-\mu \mathrm{m}$-wide straight channel with one inlet and one outlet reservoir. The control layer had two independently controlled membranes, through one central wide "trapping" channel and two narrow "isolating" channels. The trapping membrane is $2 \mathrm{~mm}$ wide to immobilize animals while the two interconnected isolation membranes are $300 \mu \mathrm{m}$ wide to keep the worm within the restricted area of the flow channel. The circular pads for punches and fluidic connections are $2 \mathrm{~mm}$ in diameter. Two photomasks were used to produce SU8 masters using UV photolithography for the flow and control layers. The flow layer was fabricated using SU8-2025 (or SU8-2050) spun at $500 \mathrm{rpm}$ for $5 \mathrm{~s}$ and $2000 \mathrm{rpm}$ for $30 \mathrm{~s}$ to obtain thicknesses of $\sim 40 \mu \mathrm{m}$ (or $\sim 80 \mu \mathrm{m}$ ). The control layer was created using SU8-2050 spun at $500 \mathrm{rpm}$ for $5 \mathrm{~s}$ and $2000 \mathrm{rpm}$ for $30 \mathrm{~s}$ to produce a thickness of $\sim 80 \mu \mathrm{m}$. PDMS (10:1) was prepared by mixing the PDMS base with the curing agent. The PDMS mix was spin coated on the flow layer at $1000 \mathrm{rpm}$ for $35 \mathrm{~s}$ to produce $\sim 120 \mu \mathrm{m}$ thickness on the SU8 master and baked at $70^{\circ} \mathrm{C}$ for $2 \mathrm{~h}$. PDMS (10:1) was poured on the SU8 pattern for a 5-mmthick PDMS mold for the control layer and baked at $70^{\circ} \mathrm{C}$ for $2 \mathrm{~h}$. The baked control layer was cut and removed from the SU8 master. Access holes were punched for trapping and isolating channels and bonded on top of the flow layer using $18 \mathrm{~W}$ air plasma for $2 \mathrm{~min}$. The bonded block was finally removed from the silicon substrate, holes were punched through for both inlet and outlet access, and bonded to a cover glass of thickness $170 \mu \mathrm{m}$.

\section{C. elegans strains}

We used the $C$. elegans transgenic strains: js/s821 [mec-7p::GFP::RAB-3] (Kumar et al., 2010) and js/s609 [mec-7p::MLS::GFP] (Fatouros et al., 2012; Sure et al., 2018) in this study. The strain $j s / s 821$ expressed GFP:: RAB-3, marking SVs of the six TRNs. For time-lapse mitochondria imaging, we used js/s609 strain expressing GFP targeted to the mitochondrial matrix in the six TRNs. Strains were maintained on NGM plates using standard protocols (Brenner, 1974). Eggs were transferred to fresh NGM plates and allowed to hatch for $2 \mathrm{~h}$ at $22^{\circ} \mathrm{C}$. Longterm imaging and mitochondrial transport parameters were calculated at multiple time points up to $36 \mathrm{~h}$ after worms were placed in the device.

\section{C. elegans maintenance inside PDMS device}

The flow layer of the device was filled with M9 buffer through the inlet and outlet. The two channels in the control layer were preconditioned with a buffer column under $14 \mathrm{psi}$ pressure of nitrogen gas. When pressurized with compressed nitrogen gas, the column of liquid did not leak into the flow channel because of the presence of the PDMS membrane. A single egg or an early larval stage animal, growing on NGM plates, was pushed into the flow channel within the region between the two isolating channels. The two isolating membranes were turned on while the central trapping membrane was left free. The OP50 bacteria culture was diluted in S medium $\left(1 \times 10^{8} \mathrm{cell} / \mathrm{s} / \mathrm{ml}\right)$, filled with $200 \mu \mathrm{l}$ microtips, and connected to the inlet of the flow channel. The outlet was connected with the second pipette tip with a lower food volume compared with the first pipette tip at the inlet. The difference in the height of the food solutions connected to the inlet and outlet channels maintains a constant flow through the flow channel. The small overlap in an area between the isolating channels and the flow channel creates a partially closed membrane in the flow channel. The partially closed channel allowed bacteria to flow along the side of the walls of the flow channel while preventing the animal from crossing the isolating membranes. A wide central trapping membrane sealed the flow channel completely under 
similar gas pressure and immobilized animals completely for high-resolution imaging.

\section{Measuring body length and diameter}

Individual animals growing inside the microfluidic devices and on NGM plates were imaged using a brightfield microscope (Olympus IX71) at $4 \times$ and $10 \times$ magnification. Images were loaded in Fiji (http://fiji.sc/Fiji) and individual worms, with dark features clearly visible on a light background, were traced with segmented lines. The segmented line was drawn from head to tail along the middle of the worm body to measure and quantify body length. The diameter was approximated with a straight line across the body in the vulva region as the maximum body width.

\section{Time-lapse imaging of $C$. elegans neurons}

C. elegans growing on NGM plates were immobilized using either $3 \mathrm{mM}$ levamisole on $2 \%$ agarose pads or without any anesthetic inside the microfluidic device under the trapping membrane for high-resolution fluorescence imaging. To count the total number of mitochondria at different developmental stages, we immobilized animals at different stages on an agarose pad using $3 \mathrm{~mm}$ sodium azide (Sigma-Aldrich) and imaged them within $10 \mathrm{~min}$ after the application of the anesthetic. Timelapse fluorescence images were acquired using a $60 \times$ (oil immersion and the numerical aperture of 1.4) objective on an inverted microscope (Olympus IX81) equipped with a spinning disk unit (CSU Yokogawa) and a CCD-based camera (iXon, Andor) to visualize GFP fluorescence from C. elegans neurons. Long-term imaging during development was conducted on the same neuron using multiple images of the same neuron at different time points. To maintain animal physiology during long-term imaging experiments, animals were left free inside the flow channel in the presence of sufficient bacteria between successive imaging sessions. To estimate synapse growth, GFP::RAB-3 fluorescence was collected from the ventral synapses of the posterior lateral mechanosensory (PLM) neurons in device immobilized or $3 \mathrm{mM}$ levamisole anesthetized animals at different stages of their development.

\section{Bleach and recovery of stationary mitochondria}

C. elegans were mounted on a glass slide on a $2 \%$ agarose pad and anesthetized with $3 \mathrm{mM}$ levamisole (Sigma-Aldrich) for live imaging. An Olympus IX83 inverted microscope attached with a spinning disk (PerkinElmer Ultraview) was used to acquire time-lapse images of the $60 \mu \mathrm{m}$ length of the initial (first $1 / 3$ from the cell body) or middle (2/3 from the cell body) of the PLM neuronal process at either 3 or 4 frames per second (fps) using a $60 \times, 1.4 \mathrm{NA}$ oil immersion objective. The Andor (iXon DU897-UVB)/Hamamatsu (SZK) monochrome camera was used to capture transport movies from GFP-labeled mitochondria for 20 min using a 488$\mathrm{nm}$ excitation laser at $\sim 8 \%$ transmission. At least 100 frames (25 s) of time-lapse movies were acquired to identify stationary mitochondria before bleaching one of the stationary mitochondria in the frame using a $405 \mathrm{~nm}$ laser at $60 \%$ transmission for $100 \mathrm{~ms}, 49$ iterations of $10 \mathrm{~ms}$ each. Kymographs of the simultaneous bleaching and imaging movies were used to identify all the stationary bleached, stationary unbleached, and mobile mitochondria.

\section{Image analysis and quantitation}

The physical dimensions of the $C$. elegans body were quantified using brightfield images of animals growing on NGM plates, in 96-well plate liquid cultures, and inside microfluidic devices. Fluorescence images were analyzed in Fiji. For mitochondrial density measurements, multiple image frames covering the entire TRN processes were opened using Fiji. Guided by the features and faint GFP signal present in the neuronal process, the length of the neuronal process was traced between successive mitochondria pairs using both the segmented and freehand line tools in Fiji. Because of linear geometry and absence of 3D curvature in the PLM neuronal process, both tools provided very similar length measurements. Hence, we used the segmented line tool for all our analyses. Every mitochondrial position and intermitochondrial intervals were recorded for every individual TRN at specified time points. Numerous total mitochondrial fluorescence envelopes were counted in a given neuronal process from the cell body to the distal tip of the process at each time point. Each fluorescence envelope was segmented to cover all the bright pixels to estimate the average intensity and total area for each mitochondrion. Intermitochondrial distances were quantified in pixels as distances between the centroid of two successive mitochondria in a neuronal process and converted to micrometers. For long-term mitochondrial analysis, the length of the neuronal process every $3 \mathrm{~h}$ was measured and plotted for an individual animal. A linear regression line was fitted to estimate the growth rate for each animal. Assuming a uniform growth rate, the length of the neuronal process at every $3 \mathrm{~h}$ time point was normalized to match the estimated value, the position of all mitochondria was corrected to match the total estimated neuronal process, and new intermitochondrial distances were calculated for further analyses.

Fast time-lapse fluorescence images of the mitochondria were converted to kymographs using the kymograph plugin in Fiji to quantify mitochondrial dynamics along the neuronal process. Kymographs were analyzed for stationary and moving mitochondria using line tracing. The anterograde and retrograde movements were determined using a minimum displacement cutoff of three pixels $(0.6 \mu \mathrm{m})$ over five consecutive frames $(1.67 \mathrm{~s})$ and a velocity cutoff of $>0.01 \mu \mathrm{m} / \mathrm{s}$. Flux was calculated, using the total number of moving mitochondria normalized to the length of the movie and the entire visible neuronal process length. The fluorescence-decay profiles of stationary mitochondria were calculated by drawing an envelope around the stationary mitochondria before bleaching and the integrated intensity profiles at all successive frames prebleach and postbleach were quantified. To normalize variation in the background because of fluctuations in the laser intensity, we calculated the background signal from an area similar in size as the 
bleached mitochondrion within the worm but away from the neuronal process and subtracted it from the integrated values. The subtracted intensity profile was used to calculate the percentage drop and the photobleaching time for each stationary bleached mitochondrion. To calculate flux across bleached and unbleached mitochondria, anterogradely and retrogradely moving mitochondria were identified at locations with stationary mitochondria and normalized to the total time elapsed during each movie.

The size of SV accumulation at ventral synapses was calculated from the total number of pixels present inside the fluorescence envelope, consisting of all the pixels with fluorescence intensities higher than the background.

\section{Statistical analysis}

Data are presented as the mean \pm SD (or SEM). Histograms are used to show the intermitochondrial distances, gain insight into the shape and variability present in the data, and estimate probability distributions from the experimental values. We used the Freedman-Diaconis rule to estimate the bin size from the interquartile range (IQR) and the number of $(n)$ intermitochondrial observations (Freedman and Diaconis, 1981). The bin width was estimated using width $=\frac{2 \times I Q R}{\sqrt[3]{n}}$, where the $I Q R$ is defined as the difference between the third and the first quartiles. To determine whether mitochondria are distributed uniformly, randomly, or clustered along the neuronal processes, we divided the neuronal processes into $20 \mu \mathrm{m}$ bins and counted the number of mitochondria in each bin. The number of mitochondria per bin, the SD of the mean, and variance $\left(\sigma^{2}\right)$ were calculated. We calculated the index of dispersion $\left(\frac{\sigma^{2}}{\mu}\right)$ for mitochondrial distribution along the neuronal processes at $t=0 \mathrm{~h}(\mathrm{~L} 4)$ and $t=24 \mathrm{~h}$ (D1) stage animals. Based on the index of dispersion, we determined whether mitochondria were distributed uniformly $\left(\mu>\sigma^{2}\right)$, randomly $\left(\mu=\sigma^{2}\right)$, or clustered $\left(\mu<\sigma^{2}\right)$. To test the goodness of fit, we used the $\chi^{2}$ analysis against a Poisson distribution (Miller and Sheetz, 2004).

The normality for experimental observations was calculated using the Kolmogorov-Smirnov test and statistical significance was calculated using either the two-sample $t$ test or one-way ANOVA (for more than two conditions). The ${ }^{*} p<0.05,{ }^{* *} p<0.005$, and $p>0.5$ (ns, not significant) was used to assess statistical significance.

\section{Results}

\section{Mitochondrial number along the neuronal process increases during development in C. elegans TRNs}

Mitochondria are essential organelles involved in energy metabolism and play a vital role in diverse biological processes such as aging and apoptosis (Sheng, 2014; Seervi and Xue, 2015; Sun et al., 2016; Melentijevic et al., 2017). Transport and distribution of mitochondria in neuronal processes and at synapses are critical for the normal physiology of neurons (Morsci et al., 2016; Cai and Tammineni, 2017). Since C. elegans are transparent throughout their development, it is feasible to track fluorescently labeled organelles such as mitochondria and SVs in TRNs using high-resolution imaging. C. elegans TRNs are a suitable model for high-resolution in vivo organelle imaging because of its long and planar neuronal process along the body length present close to the cuticle (Fig. 1A,B).

To quantify mitochondrial density in $C$. elegans TRNs, we immobilized js/s609 animals and imaged the TRNs using mitochondrial matrix targeted GFP expressed in six TRNs for a single time point analysis during their development (Fatouros et al., 2012; Sure et al., 2018). We immobilized the animals on a $2 \%$ agarose pad using $3 \mathrm{~mm}$ sodium azide and counted mitochondria number, within $10 \mathrm{~min}$ after the application of the anesthetic, using mitochondrial GFP and length of the neuronal process visible because of some GFP that fills the neuronal process in the PLM neurons (Fig. 1C). An increase in neuronal process length has been shown to correlate with an increase in mitochondria number as the animal ages and across developmental stages (Morsci et al., 2016; Awasthi et al., 2020). We found the neuronal process length increases from $\sim 100 \mu \mathrm{m}$ in early larval stage 1 animals (L1) to nearly $500 \mu \mathrm{m}$ in 1-d adult (1D adult) with a concomitant increase in mitochondria number from $\sim 6$ to 26 in the posterior TRN (Fig. 1D). The bulk of the increase in the length of the neuronal process occurs as the animal grows and occurs after synapse formation at the early L1 stage of development. The addition of new mitochondria maintains a mitochondrial density of approximately five mitochondria per $100 \mu \mathrm{m}$ along the neuronal process length throughout development (Fig. 1E; Table 1). This density is similar to earlier reports of density measured in adult animals for this neuron (Morsci et al., 2016; Sure et al., 2018). From the above values, the increase in mitochondrial number during development is approximated to approximately six mitochondria every $24 \mathrm{~h}$ (averaging approximately one mitochondrion every $4 \mathrm{~h}$ ). The area of mitochondria fluorescence in L1, L4, and 1D adult stages of the animal is measured to vary between 0.05-0.95, 0.05-1.66, and 0.05-2.14 $\mu \mathrm{m}^{2}$, respectively (Fig. $1 F$ ). Young larval animals have a greater number of smaller mitochondria compared with later larval stages or adult animals. Single time point images of $C$. elegans populations of different developmental stages indicate a steady increase in the total number of mitochondria but fail to indicate the location of such addition. To capture individual mitochondrion addition events such that a similar mitochondria density is maintained over development, the same neuronal process needs to be imaged over days under optimal growth and imaging conditions. Since mitochondria are susceptible to cellular stresses (Hill and Van Remmen, 2014; Jovaisaite et al., 2014; Labbadia et al., 2017), it is impossible to image the same animal by repeatedly anesthetizing it over a prolonged growth period. To reduce the adverse effects of anesthetics and provide a physiological environment, we developed a long-term growth and imaging microfluidic platform.

\section{Microfluidic device facilitates growth and high- resolution imaging of $C$. elegans}

To study the long-term development of individual C. elegans while carrying out high-resolution imaging, we 
A

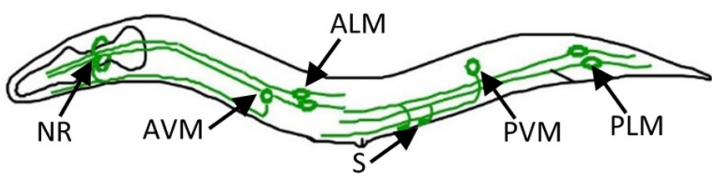

C
B

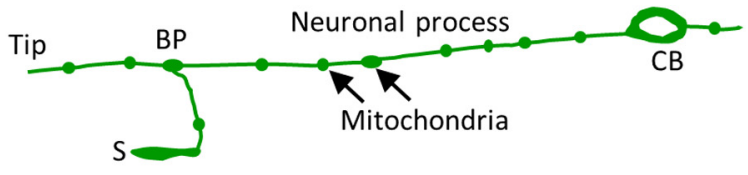

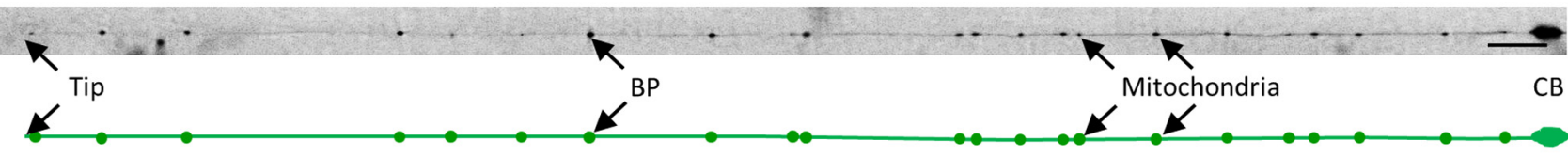

D

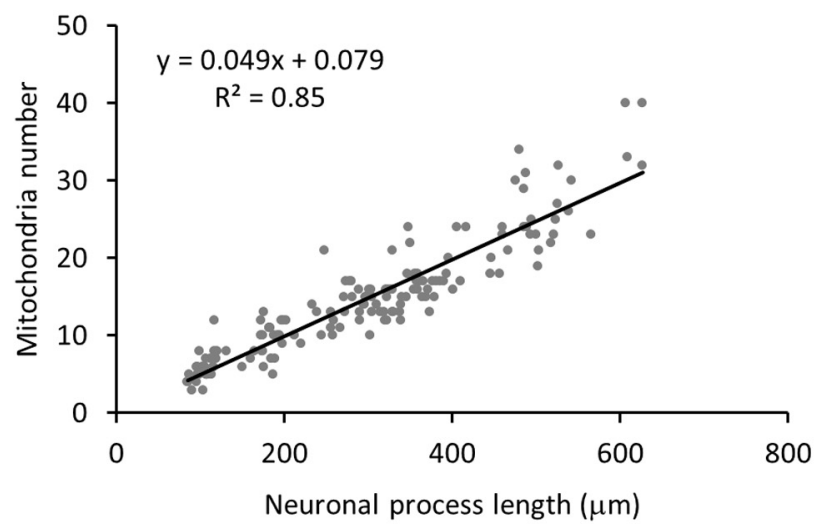

E

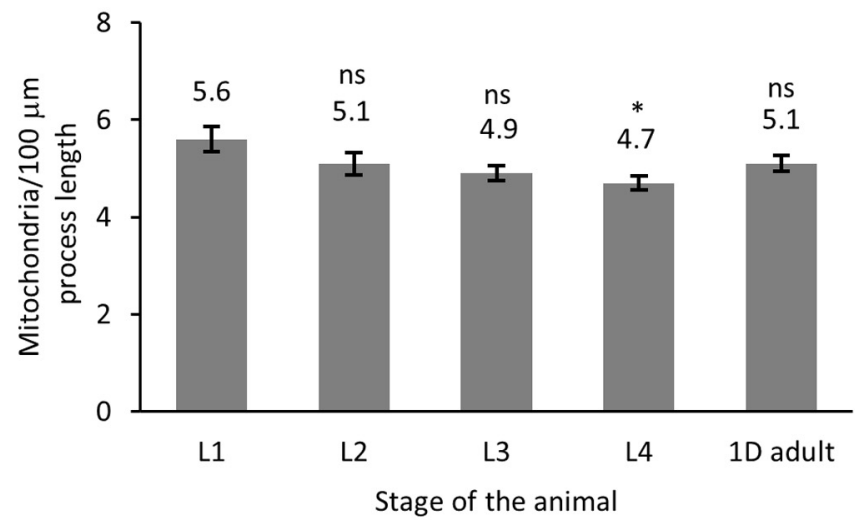

$\mathbf{F}$

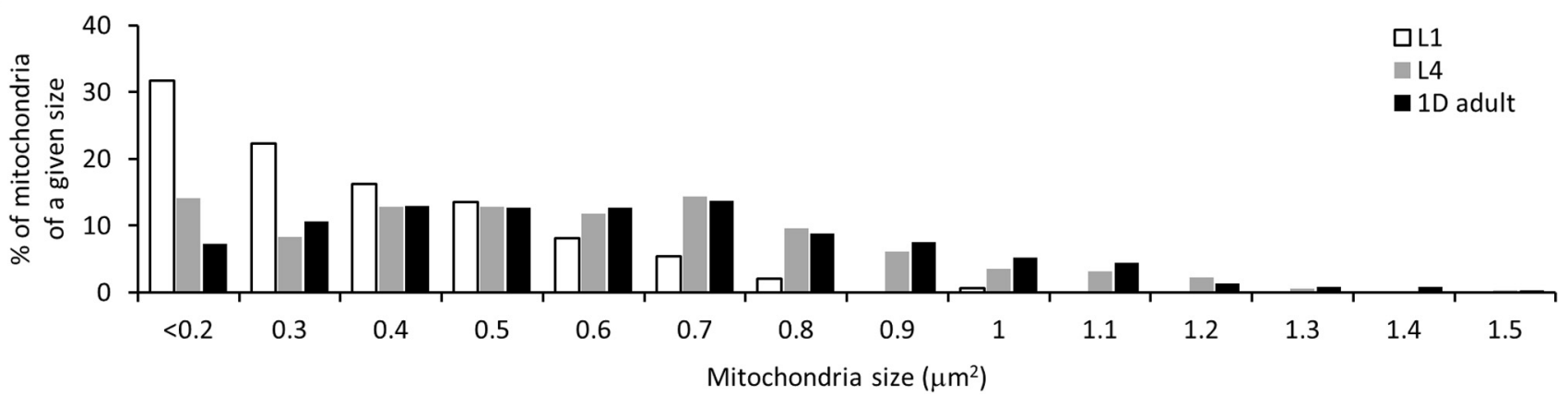

Figure 1. Mitochondria number and process length increase linearly with development. $\boldsymbol{A}$, Schematic of $\boldsymbol{C}$. elegans with six TRNs. B, Schematic of PLM neuron cell body (CB) with a long major neuronal process with a bifurcation of the process at the BP to form the synaptic branch that forms synapses (S) along the ventral cord. The mitochondria are indicated with arrows. C, Image of a PLM neuron with 22 mitochondria along the neuronal process. The schematic below the image shows the positions of all 22 mitochondria in the same neuronal process. Scale bar: $20 \mu \mathrm{m}$. $\boldsymbol{D}$. Mitochondria number scales linearly with neuronal process length. Neuronal process length and number of mitochondria of all four larval stages (L1, L2, L3, and L4) and 1D adult. The trend follows a linear regression with a slope of 4.9 mitochondria in $100-\mu \mathrm{m}$ neuronal process length (total number of animals $=154)$. $E$, Mitochondria density remains constant throughout development. Data represented as mean \pm SEM for L1 $(n=30)$, L2 $(n=25)$, L3 $(n=36)$, L4 $(n=33)$, and 1D adult $(n=30)$. Statistical significance was evaluated using one-way ANOVA with Bonferroni post hoc comparisons; ${ }^{*} p<0.05$ and $\mathrm{ns}, p>0.05$ when compared with L1 stage. $\boldsymbol{F}$, Histogram of the normalized number of mitochondria of a given size represented as a \% in the TRNs of L1, L4, and 1D adult stage of animals ( $n \geq 148$ mitochondria for each stage).

developed a microfluidic device to mimic a physiological environment by supplying constant food where we can immobilize the animal under a deformable PDMS membrane as needed. To make this microfluidic technology easily accessible, we developed a device with a simple design, few fabrication steps, easy operation, no complex valves, using inexpensive accessories. The PDMS device is bonded on a thin cover glass to facilitate high-resolution imaging on an inverted microscope using lenses of various magnifications up to a $100 \times$ oil objective.
The device is fabricated as two separate PDMS layers and irreversibly bonded together (Fig. 2A-F). The device utilizes PDMS membrane deflections to isolate and immobilize a $C$. elegans hermaphrodite within the flow channel. In this device, $C$. elegans swim freely and grow in a $300-\mu \mathrm{m}$-wide flow channel in the presence of bacteria. A pair of partially closed membranes above the symmetric isolating channels allows the bacterial suspension to pass through while preventing an individual animal from escaping the flow channel. The individual animal is repeatedly 
A

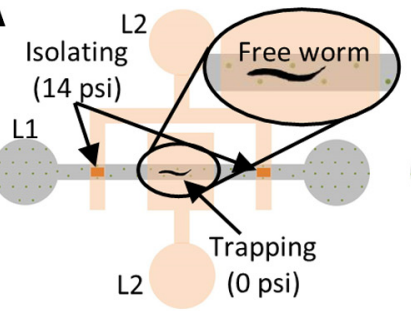

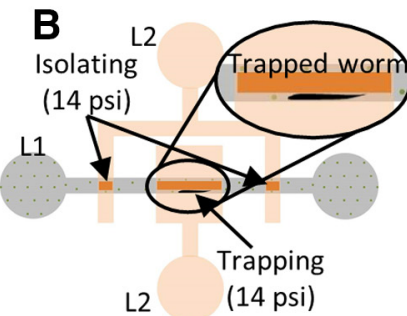

E

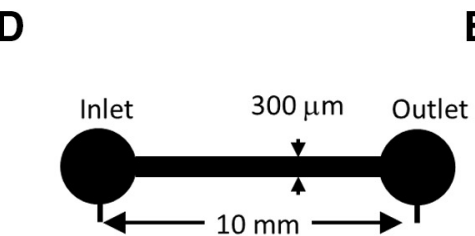

G
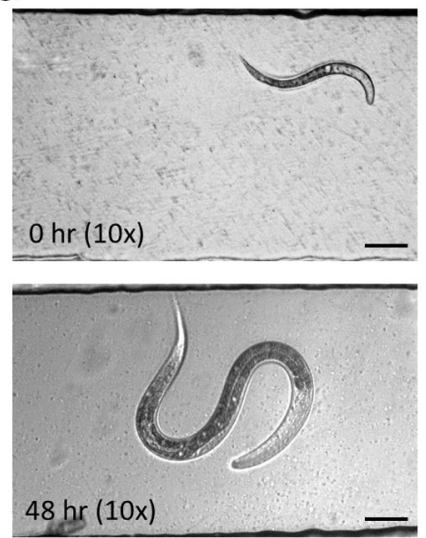
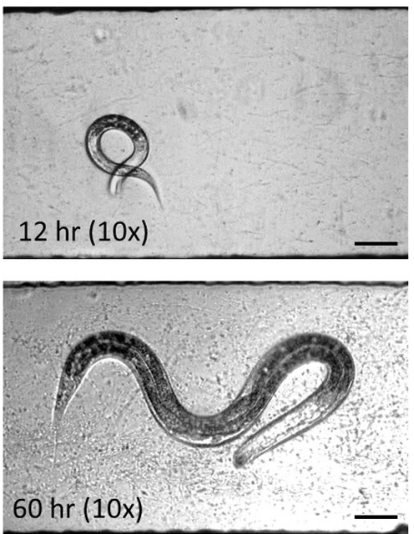

H

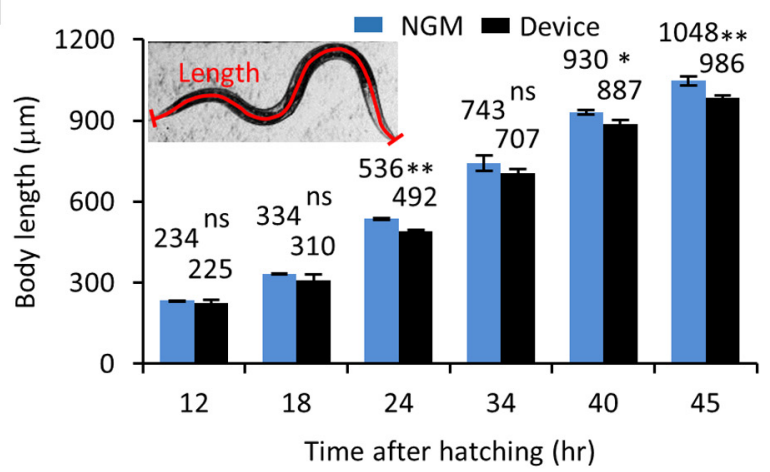

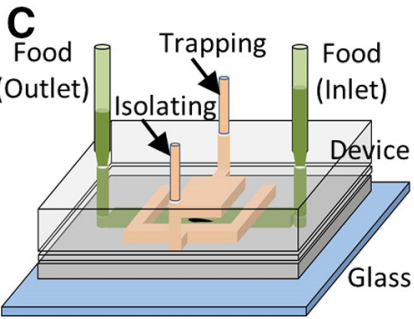
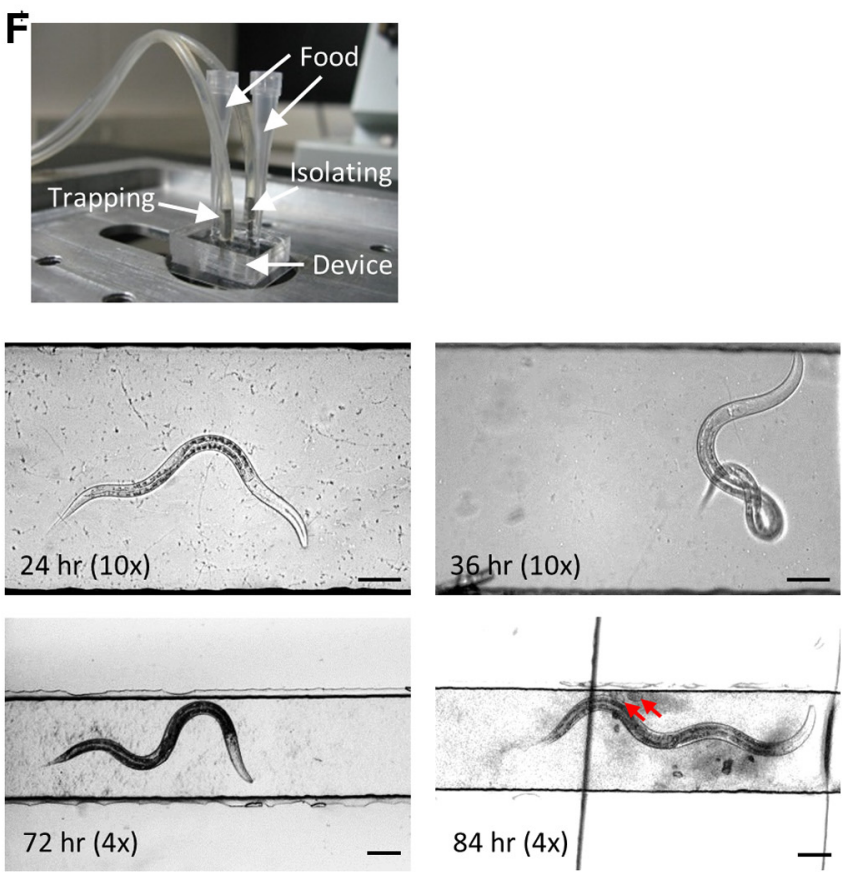

I

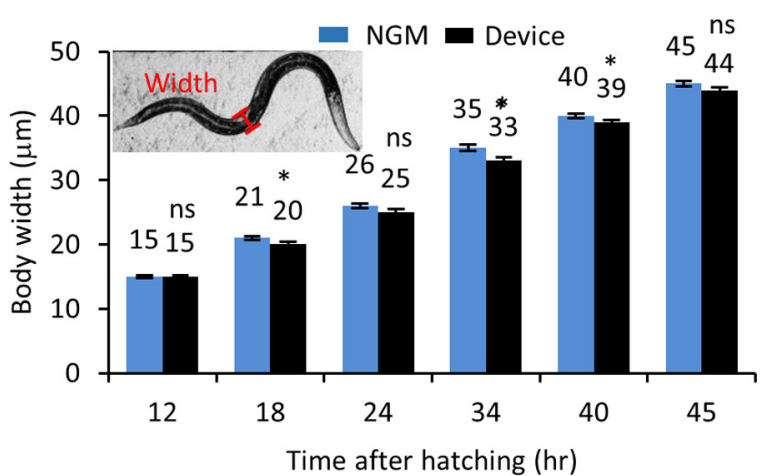

Figure 2. Growth and development of $C$. elegans are unaffected in the long-term growth and imaging microfluidic device. $\boldsymbol{A}$, Schematic of the microfluidic device with a flow channel in the bottom layer (L1, gray) and isolating and trapping channels in the control layer on top (L2, orange). The isolating membrane is always kept on, with 14 psi pressure (deflected membrane denoted by dark orange) to restrict the free animal shown in the inset. $\boldsymbol{B}$, Trap pressure is turned on (14 psi, deflected membrane denoted by dark orange) to immobilize the animal inside the flow channel (inset) during imaging. $\mathbf{C}$, 3D view of the device layout with two pipettes tips (light green) as food inlet and outlet connecting the flow channel (dark green). $\boldsymbol{D}$, Schematic for the flow layer with channel dimensions. $\boldsymbol{E}$, Schematic of the control layer with channel dimensions. $\boldsymbol{F}$, Image of a growth and imaging device connected to compressed nitrogen gas and food supply. G, Images of a $C$. elegans hermaphrodite growing inside the microfluidic device at 0,12 , $24,36,48,60,72$, and $84 \mathrm{~h}$ posthatching. The animal is fed OP50 bacteria and isolated inside the flow channel and imaged with a $4 \times$ or $10 \times$ objective. The red arrows indicate freshly laid eggs at $84 \mathrm{~h}$. Scale bars: 50 or $100 \mu \mathrm{m}$ at the $72-$ and $84-\mathrm{h}$ time points. $\boldsymbol{H}$, $\boldsymbol{I}$, js/s609 animals grown in the device (black) and on regular NGM plates (blue) were used for calculating body length $(\boldsymbol{H})$ and body diameter $(\boldsymbol{l})$. The small inset shows the schematic for body length and body diameter from worm images. The average values are mentioned on top of each bar. Data represented as mean \pm SEM, $n=12$ animals. Statistical significance was evaluated by paired sample $t$ test; ${ }^{*} p<0.05,{ }^{* *} p<0.005$, and ns, $p>0.05$ when compared between animals grown on NGM and in liquid culture. 
immobilized under a deflected "immobilizing" PDMS membrane. In vivo cellular processes are imaged in the animal immobilized beneath the trapping channel.

\section{Growth in a microfluidic device does not impede $C$. elegans development}

To obtain high-quality time-lapse movies of the subcellular events during $C$. elegans development, the health of the animal grown in a microfluidic environment during the entire period should be similar to that of an animal grown on standard NGM solid media. C. elegans growth in liquid medium with Escherichia coli bacteria has been studied (Solis and Petrascheck, 2011; Keil et al., 2017). C. elegans were found to develop $\sim 15 \%$ slower in liquid culture with adequate bacterial supply nonetheless recapitulated all long-term developmental processes such as molting and egg laying (Keil et al., 2017). Since food supply is one critical component for normal development, we ensured sufficient bacterial supply by adjusting both the concentration of bacteria and the relative amounts of buffer solution with bacteria in the two pipette tips attached to the channel entrances. The flow channel containing liquid OP50 showed no contamination over $84 \mathrm{~h}(4 \mathrm{~d})$ of $C$. elegans growth (Fig. 2G). The pipette tips can be easily replaced with new sterile tips freshly filled with OP50 bacteria as needed.

To characterize $C$. elegans development in our microfluidic device, we measured the body length and diameter of the animals grown in our microfluidic chamber and compared them to values obtained from animals grown on NGM solid media. Our data show that animals growing in the microfluidic device are on average shorter and thinner compared with those grown on solid NGM media (Fig. $2 H, l ;$ Table 1). The body features (body length and diameter) of the animals grown inside the microfluidic device lags by $2-3 \mathrm{~h}$ compared with the values obtained from those grown on NGM solid media. These data are similar to those reported earlier for $C$. elegans grown in liquid media (Solis and Petrascheck, 2011; Keil et al., 2017). A hermaphrodite grown inside the microfluidic device laid eggs after $84 \mathrm{~h}$, that hatch into larvae.

\section{Microfluidic device facilitates repeated immobilization and time-lapse imaging of the identical neuronal process in an individual animal}

Mitochondria in the neuronal process display distinct transport characteristics; characteristic velocities away from (anterograde) and toward (retrograde) the cell body, intermittent pauses, and bi-directional movement (Welte, 2004; O'Toole et al., 2008; Cai and Sheng, 2009; Schwarz, 2013; Hancock, 2014; Sure et al., 2018). To quantify in vivo mitochondrial transport characteristics from the C. elegans TRN processes, we immobilized animals in the flow channel using 14 psi on the trapping PDMS membrane (Fig. 3A). A slow progressive immobilization allows animals to be laterally oriented, facilitating high-resolution imaging of PLM neurons.

To assess the effect of photobleaching and changes in auto-fluorescence because of repeated immobilization, we repeatedly immobilized $C$. elegans at two different time intervals; either trapping every $1 \mathrm{~h}$ or trapping every $6 \mathrm{~h}$ under the immobilizing membrane up to a total duration of $12 \mathrm{~h}$. Under both paradigms, the same PLM neuron (either $L$ or R) of the animal was imaged only at the 0-, 6-, and 12-h time points. The ratio of the mitochondrial fluorescence to the background fluorescence (M/B ratio) was calculated at different time points. The decrease in this fluorescence ratio in both paradigms was statistically insignificant $(2.07 \pm 0.106,1.80 \pm 0.192, p=0.06$; Extended Data Fig. $3-1 A-C$ ). Using repeated imaging of $C$. elegans in our microfluidic device, we were able to obtain a good fluorescence signal from mitochondria compared with the background autofluorescence.

Individual animals of the appropriate developmental stage were picked from an NGM plate, using $5 \mu \mathrm{l}$ M9 buffer, and inserted inside the flow channel in the absence of immobilization or isolation pressure. The animal inside the flow channel was observed using either a $4 \times$ or a $10 \times$ objective on an inverted microscope. The height of the bacterial solution in the two pipette tips, connected at the inlet and outlet, generated sufficient hydrodynamic flow rates to allow bacterial passage through the flow channel to feed the worm (Fig. 2C,F). The deflected membrane in the isolating channel restricts animal motion between the two isolating channels. Freely moving worms were imaged in brightfield to measure their physical parameters and assess animal health. Animals were completely immobilized in the flow channel under the PDMS membrane using 14 psi pressure in the trapping channel. Complete immobilization was required for high-resolution timelapse imaging of the subcellular events in the neuronal processes. Animals were immobilized repeatedly in a straight posture along the flow channel sidewalls under the trapping membrane to bring the same posterior lateral TRN under the best focus for imaging the subcellular events (Fig. 3A). Identical animal orientation enabled us to image mitochondrial distribution from the same individual neuron over multiple time points using an inverted confocal microscope equipped with a $60 \times$ oil objective (numerical aperture 1.4).

We further characterized the effect of immobilization of an individual $C$. elegans on the variability in the position of each stationary mitochondrion that arises just from the immobilization process and small changes in the posture of the animal. Positions of mitochondria were used to calculate intermitochondrial distances and estimate local compression and expansion of the neuronal process between multiple adjacent mitochondrial pairs. We, therefore, imaged the same TRNs every $5 \mathrm{~min}$ for $15 \mathrm{~min}$ by repeatedly trapping the animal under the immobilizing PDMS membrane and calculated intermitochondrial intervals for each immobilization. Intermitochondrial distance values $(L)$ between identical mitochondrial pairs from the same TRN processes imaged at different time points were compared $(\Delta \mathrm{L})$. This comparison allows estimation of how the extent of expansion or compression between each mitochondria pair $(\Delta \mathrm{L} / \mathrm{L})$ affects adjacent intermitochondrial distances. Only stationary bright mitochondria were considered for this calculation. Most mitochondrial 
A

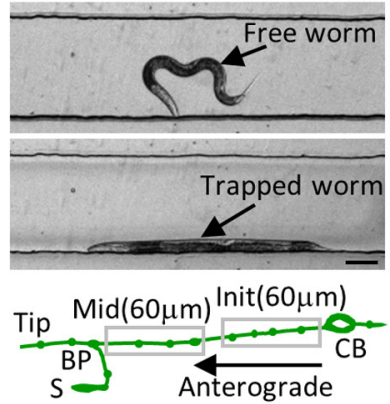

E

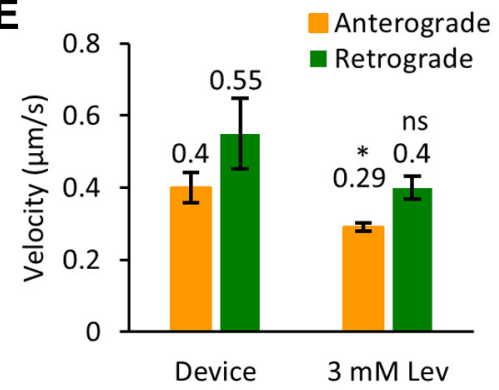

B

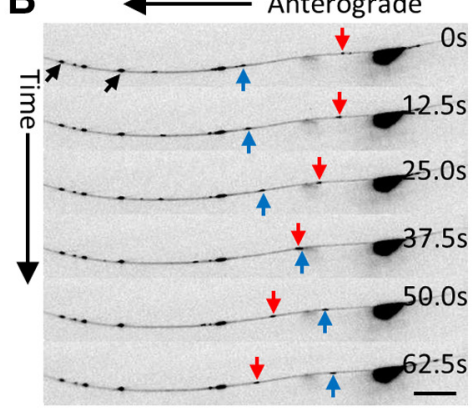

C

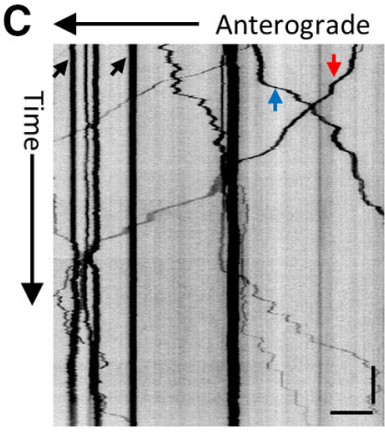

D

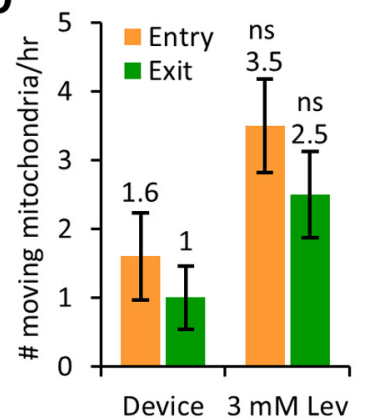

$\mathbf{F}$

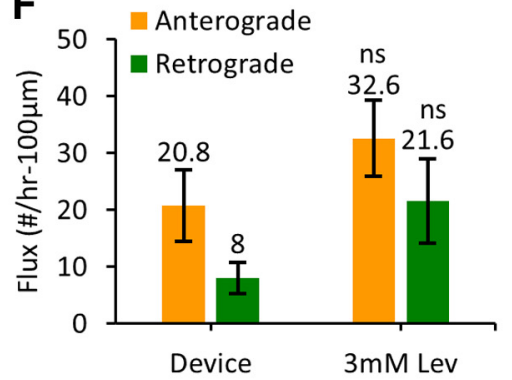

G

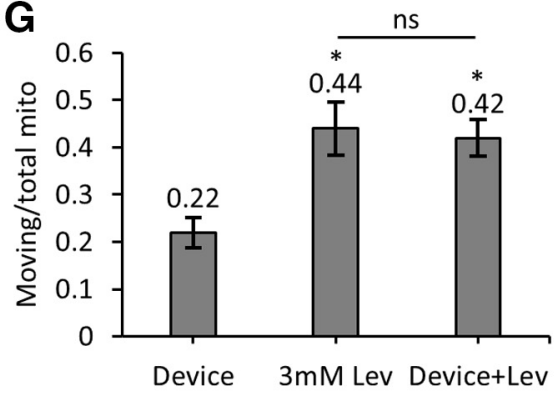

H

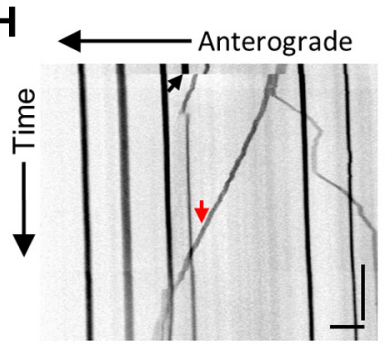

Anterograde (Ant) cross

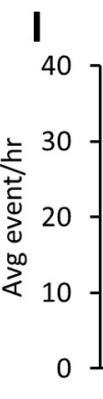

0

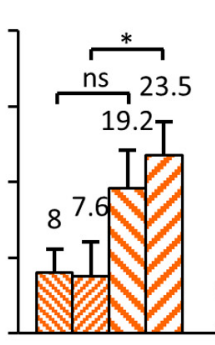

Ant cross

QDevice(Init) 口Device(Mid) $\checkmark$ Levamisole(Init) Levamisole(Mid)

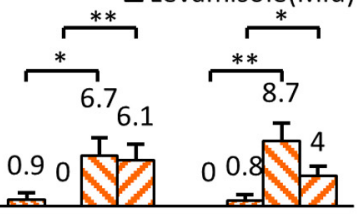

Ant pause

Ant split

$\mathbf{L}$

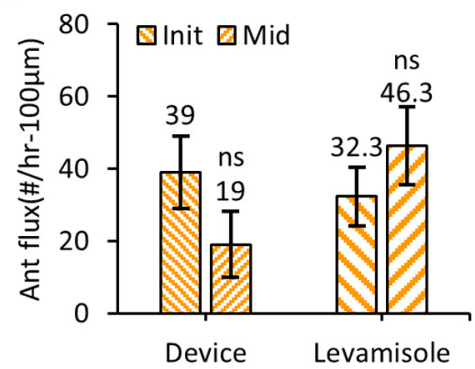

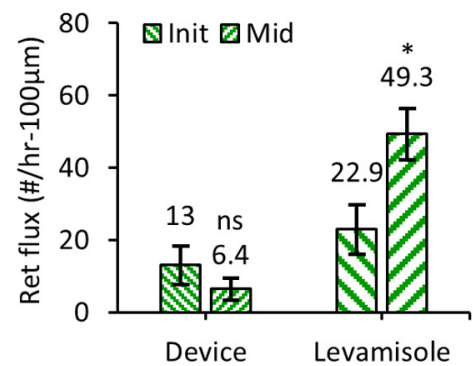

J

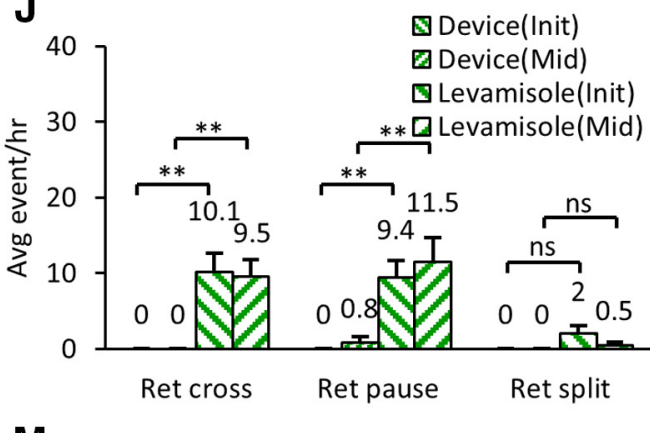

M

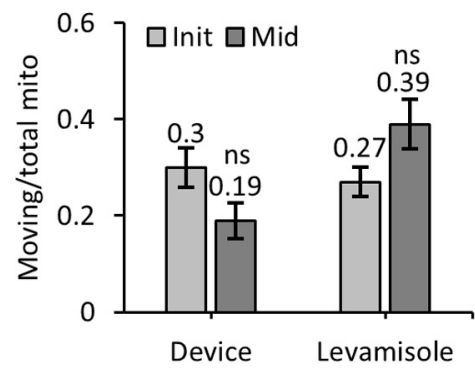

Figure 3. High-resolution transport imaging of GFP-labeled mitochondria in levamisole and device immobilized C. elegans neurons. $\boldsymbol{A}$, Image of a free and an immobilized L4 stage $C$. elegans in the flow channel. The schematic shows the initial $60 \mu \mathrm{m}$ from the cell body and the middle of the neuronal process $(120 \mu \mathrm{m}$ from the BP toward the cell body) that is imaged at high resolution. $\boldsymbol{B}$, Montage of six frames of a PLM neuron with GFP-labeled mitochondrial fluorescence from time-lapse imaging acquired using a $60 \times$ oil objective (1.4 NA). C, Kymograph of 400 frames acquired at 2 fps for 200 s. Anterogradely moving (red arrow), retrogradely moving (blue arrow), and stationary (black arrow) mitochondria are indicated on the images. $\boldsymbol{D}$, Number of mitochondria entering the neuronal process (entry) and leaving the process (exit) per hour measured in animals immobilized with $3 \mathrm{mM}$ levamisole ( $n=35$ animals) and inside the microfluidic device ( $n=22$ animals). $\boldsymbol{E}$, The average velocities (both in anterograde and retrograde directions) are shown for animals that are immobilized inside the microfluidic device ( $n=47$ anterograde and $n=15$ retrograde segments) and compared with animals immobilized with $3 \mathrm{mM}$ levamisole $(n=336$ anterograde and $n=142$ retrograde segments). $\boldsymbol{F}$, The average flux values of moving mitochondria from L4 stage animals immobilized inside the microfluidic device $(n=16)$ and with 3 mM levamisole $(n=18)$. $G$, The plot showing the ratio of moving mitochondria to the total number of mitochondria for device immobilized $(n=16)$, levamisole immobilized $(n=18)$, and levamisole treated animals that are also immobilized in the device $(n=14)$. $\boldsymbol{H}$, Kymograph representing an anterograde moving mitochondrion (red arrow) crossing a site of a bleached stationary mitochondrion (black arrow). I, The average anterograde events per hour (cross, pause, and split) are measured at the initial (Init; $60 \mu \mathrm{m}$ from the cell body) and the middle (Mid; a region $120 \mu \mathrm{m}$ from the BP toward the cell body) of the neuronal process (schematic is shown in 
continued

panel $\boldsymbol{A}$ ) for both device (Device; $n>8$ animals) and anesthetic (Levamisole; $n>29$ animals) immobilized L4 animals. $\boldsymbol{J}$, The average retrograde events are measured by using the device and anesthetic immobilized animals. $\boldsymbol{K}$, The anterograde flux are measured from all the moving mitochondria in the device and levamisole immobilized L4 animals from the Init and Mid of the PLM neuronal process. $\boldsymbol{L}$, The retrograde flux are measured from all the moving mitochondria in the device and levamisole immobilized $L 4$ animals from Init and Mid neuronal processes. Anterograde and retrograde fluxes are calculated from $n=10$ (Init) and $n=10$ (Mid) animals in the device, while $n=16$ (Init) and $n=26$ (Mid) animals in the levamisole. $M$, The ratio of moving mitochondria to the total number of mitochondria for the Init and Mid portion of the PLM in the device and levamisole immobilized animals. Data represented as mean $\pm \operatorname{SEM}(\boldsymbol{D}, \boldsymbol{E}-\mathbf{G}, \boldsymbol{I}-\boldsymbol{M})$. Statistical significances are evaluated by paired sample $t$ test $(\boldsymbol{D}-\boldsymbol{F}$, $\boldsymbol{I}-\boldsymbol{M})$ and one-way ANOVA $(\boldsymbol{G})$; ${ }^{*} p<0.05,{ }^{* *} p<0.005$, and ns, $p>0.05$ are represented. Scale bars: $100 \mu \mathrm{m}(\boldsymbol{A}), 10 \mu \mathrm{m}(\boldsymbol{B}, \boldsymbol{H})$, and $5 \mu \mathrm{m}(\boldsymbol{C})$. Vertical bars: 20 s $(\boldsymbol{C}$, H). Table 2, Extended Data Figures 3-1, 3-2, and Movies 1, 2, 3 support this figure.

positions remain largely unchanged and intermitochondrial distances were used to measure the compression or expansion percentages between each pair of mitochondria (Extended Data Fig. 3-1D,E). The compression and expansion percentages were distributed symmetrically and represented as positive (compression) and negative (expansion) values, respectively. The $\Delta \mathrm{L} / \mathrm{L}$ values were distributed around $+1.79 \%$ and $-2.01 \%(2.4 \pm 0.17$ and $2.4 \pm 0.19 \mu \mathrm{m}$, mean \pm SEM; Extended Data Fig. 3-1E). Thus, the errors arising from compression and expansion because of immobilization do not significantly alter the measurement of intermitochondrial distances.

\section{Device-immobilized animals show lower mitochondria flux and turnover compared with anesthetic immobilized animals}

We examined mitochondria numbers and their dynamics in NGM grown and device grown animals. The density of mitochondria in larval stages and 1D adult animals grown in NGM and microfluidic devices are similar.

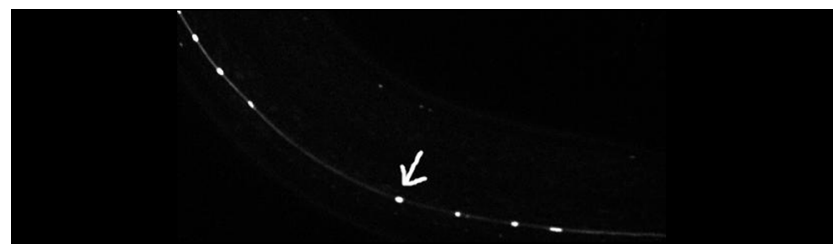

Movie 1. Time-lapse movie showing a cross event across a bleached stationary mitochondrion indicated by the arrow. [View online]

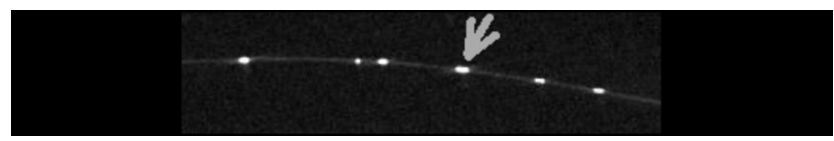

Movie 2. Time-lapse movie showing a pause at the location of a bleached stationary mitochondrion, indicated by the arrow. [View online]

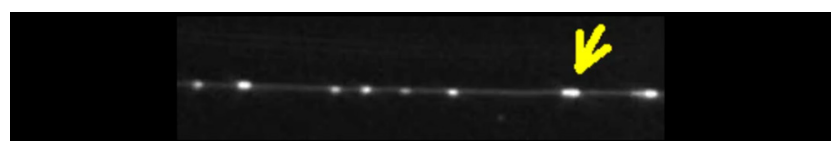

Movie 3. Time-lapse movie showing a split event at the location of a bleached stationary mitochondrion, indicated by the arrow. [View online]
Additionally, the intermitochondrial intervals in NGM grown animals and device grown animals are very similar. To examine the movement and turnover of mitochondria from both device and anesthetic immobilized animals, we conducted high-resolution time-lapse imaging of TRNs. The acquired movies were converted into kymographs to analyze mitochondrial transport and positions (Fig. $3 B, C)$. Device immobilized animals regained normal body movements within $10 \mathrm{~min}$ of release from pressure. Mitochondria are classified as stationary if their displacement is less than three pixels $(0.6 \mu \mathrm{m})$ over five consecutive frames $(1.67 \mathrm{~s})$ or their velocity is $<0.01 \mu \mathrm{m} / \mathrm{s}$. A mitochondrion is considered as moving if its displacement is more than or equal to three pixels over five consecutive frames. Mitochondria move anterogradely away from the cell body or retrogradely toward the cell body. The movement close to the cell body was captured for $20 \mathrm{~min}$ at $3 \mathrm{fps}$ to quantify all anterograde and retrograde events in both anesthetized animals and device immobilized L4 stage animals, both grown on NGM plates until imaged. The average number of mitochondria entering the neuronal process from the cell body was about twice the total number of mitochondrial exit events into the cell body (Fig. 3D). The average number of entry and exit events was lower but not statistically different $(p>0.5)$ in the device immobilized animals compared with anesthetized animals (Table 1). In device immobilized animals, the rate of mitochondrial entry into the process is twice that of mitochondrial exit as well (Fig. 3D).

The average instantaneous velocity of moving mitochondria in both the anterograde and retrograde directions was slightly higher in the device $(0.40 \pm 0.042$ and $0.55 \pm 0.098 \mu \mathrm{m} / \mathrm{s}$ ) compared with anesthetic immobilized animals $(0.29 \pm 0.011$ and $0.40 \pm 0.031 \mu \mathrm{m} / \mathrm{s})$, although statistically significantly different only for the average anterograde velocities (Fig. 3E; Table 2). The average anterograde mitochondria flux value was higher than that of the retrograde flux in both device and anesthetic immobilized animals (Fig. 3F). The anterograde and retrograde fluxes in the levamisole immobilized animals (32.6 \pm 6.70 and $21.6 \pm 7.40$ mitochondria/h/100 $\mu \mathrm{m}$ ) were higher as compared with that in the device immobilized animals $(20.8 \pm 6.28$ and $8.0 \pm 2.74$ mitochondria/h/100 $\mu \mathrm{m})$ although the differences are not statistically significant $(p=0.20$ and $p=0.10)$. We examined whether the greater mitochondrial transport in levamisole (3 mM Lev) treated worms, compared with worms immobilized in the device without chemicals (Device) was because of the anesthetic. We acquired time-lapse images of PLM neurons 
from animals both anesthetized in $3 \mathrm{mM}$ levamisole and trapped in our microfluidic device (Device + Lev). The ratio of all moving mitochondria to the total number of mitochondria in levamisole immobilized (0.44 \pm 0.056 , $n=18$ ) animals were statistically significantly higher $(p=0.004)$ than in device immobilized $(0.22 \pm 0.032$, $n=16$ ) animals (Fig. 3G). The ratio of moving to stationary mitochondria continued to remain high $(0.42 \pm 0.039$, $n=14$ ) in levamisole anesthetized animals mechanically trapped by a PDMS membrane (Fig. 3G). Similar effects by anesthetics on neuronal transport flux of vesicular cargo have been reported (Mondal et al., 2011).

Using fast time-lapse imaging of the individual TRNs, at a speed of 3 or $4 \mathrm{fps}$ for a total of 1000 frames (time $\geq 150 \mathrm{~s}$ ), we analyzed turnover rates of moving mitochondria across photobleached stationary mitochondria in the middle of the neuron. The stationary mitochondria drop fluorescence intensity by $96.5 \pm 0.89 \%$ (from $n=20$ bleaching events) within $0.33 \mathrm{~s}$, the interval between successive frames at our acquisition rate of $3 \mathrm{fps}$ (Extended Data Fig. 3-1A-C). Upon photobleaching a stationary mitochondrion, the motion of a small and faint moving mitochondrion can be easily tracked across the site of the photobleached stationary mitochondrion. In a simple cross event, a small mitochondrion moves across the photobleached mitochondrion without contributing fluorescence to the stationary mitochondrion (Fig. $3 \mathrm{H}$; Extended Data Fig. 3-2D; Movie 1). While moving mitochondria are identified as contributing to a photobleached stationary mitochondrion by either pausing and/or splitting a portion of their fluorescence leading to fluorescence recovery at the site of the large photobleached stationary mitochondrion (Extended Data Fig. 3-2E-G; Movies 2, 3). Sometimes paused mitochondria filled the entire region of the stationary mitochondria area before photobleaching and sometimes they merely pause and then continue to move. Both anterograde and retrograde moving mitochondria were found to contribute to the turnover of stationary mitochondria. On average, more events were observed in anesthetized animals compared with device-immobilized animals across any given bleached mitochondria, likely because of the overall increase in flux in levamisole anesthetized animals (Fig. 3/,J). Both the device and levamisole immobilized animals show a higher number of anterograde movements across stationary bleached mitochondria as compared with retrograde movements, similar to that observed in movies without bleaching (Fig. 3E,F; Table 2; Extended Data Fig. 3-2H). We also measured total flux as well as a proportion of events in both the initial 1/3 (Init) and the middle (Mid) of the neuron (Fig. $3 K, L)$. We see the proportion of moving mitochondria is greater in the levamisole immobilized animal in every region of the neuron image (Fig. $3 M)$. This might account for the small differences in the number of events where a moving mitochondrion interacts with the stationary bleached mitochondria.

We did not observe any stationary mitochondria split into smaller mitochondria in any of our movies during the entire $4.6 \mathrm{~h}$ of imaging either in anesthetic or device immobilized animals. The fluorescence envelopes of moving mitochondria were smaller than stationary mitochondria. We sometimes observed tubular-shaped moving mitochondria in the first $60 \mu \mathrm{m}$ of the neuronal process proximal to the cell body. Anesthetic immobilization effects perhaps account for the increase in mitochondrial movement which in turn may lead to a higher turnover of mitochondria along the neuronal process.

\section{Mitochondria are added along the entire C. elegans TRN neuronal process}

Mitochondria are added to a growing neuron after synapse formation to maintain a constant mitochondrial density (Morris and Hollenbeck, 1993). For example, the number of mitochondria and total process length respectively increased from $7.8 \pm 0.41$ and $125 \pm 6.07 \mu \mathrm{m}$ in $\mathrm{L} 2$ larvae ( $n=15$ animals) to $20.1 \pm 1.44$ and $335 \pm 15.89 \mu \mathrm{m}$ in L4 larvae ( $n=10$ animals, $p<0.01)$ in the microfluidic device. The neuronal process grows at the rate of $\sim 10 \mu \mathrm{m} / \mathrm{h}$ and the mitochondria number increases $\sim 0.6$ mitochondria/ $h$, numbers very similar to those seen in animals grown on NGM plates (see above). Although the population statistics indicate average growth rates, it does not indicate the site where new mitochondria are added.

To identify the location where the new mitochondria are added as the animal is growing, we imaged the same PLM neuron every $3 \mathrm{~h}$ for $\sim 36 \mathrm{~h}$ by repeatedly trapping the animal in our microfluidic device. The images were used to reconstruct the neuron and the location of each mitochondrion was plotted for every imaged time point. The total mitochondria number increased linearly with imaging time. Both, total mitochondria number and neuronal process length at $24 \mathrm{~h}$ were significantly higher than at the initial time point (Fig. 4A; Table 1). We chose the cell body, branch point (BP), and the neuronal process tip as the fiduciary markers to examine whether there was any bias in the growth of the neuronal process as new mitochondria are added along the neuronal process (Fig. $4 E$ ). The ratio of the neuronal process lengths between the cell body to the BP and the BP to the neuronal process tip remains constant as the neuron and the animal grows suggesting proportional growth along the neuronal process (Fig. 4B,C). Over $36 \mathrm{~h}$ of imaging, the average total neuronal process growth rate was calculated to be $\sim 9 \mu \mathrm{m} / \mathrm{h}$ ( $n=8$ animals). We calculated the ratio of the mitochondria number present along the neuronal process between the cell body and the BP to the mitochondria number between the BP and the neuronal tip. The ratio of the mitochondria number remains constant over a $24 \mathrm{~h}$ period (Fig. 4B), which suggests that new mitochondria are likely added uniformly along the process length.

To capture the position and size of every mitochondrion, we reconstructed the entire neuronal process from a series of fluorescence images at multiple time points and analyzed various parameters such as mitochondrial number, size, and the intermitochondrial intervals between each adjacent pair of mitochondria. $\sim 20 \%$ of mitochondria move in any given frame in the TRNs with an average velocity of $\sim 200 \mathrm{~nm} / \mathrm{s}$ (Fatouros et al., 2012). Moving mitochondria are less than $\sim 1 \mu \mathrm{m}$ in diameter. Stationary mitochondria are bigger in size but drift gradually over time as the animal grows and the neuronal process commensurately proportionately elongates. Small moving 


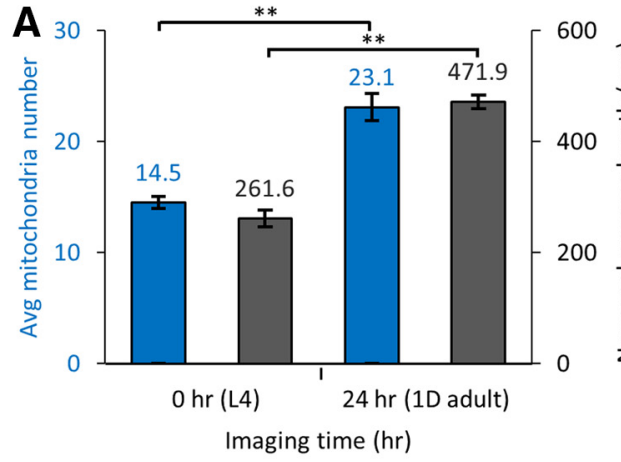

C

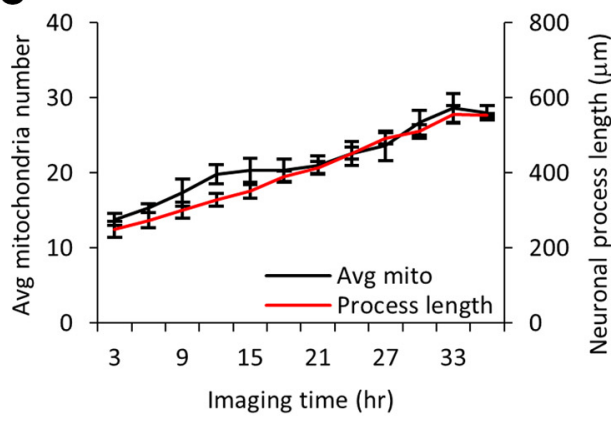

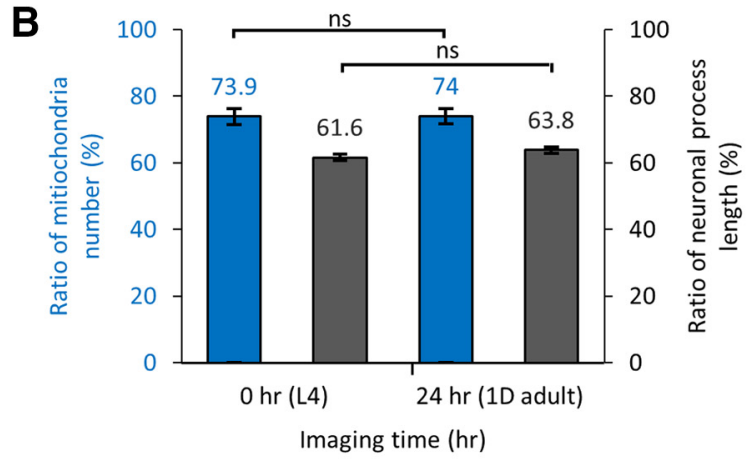

D

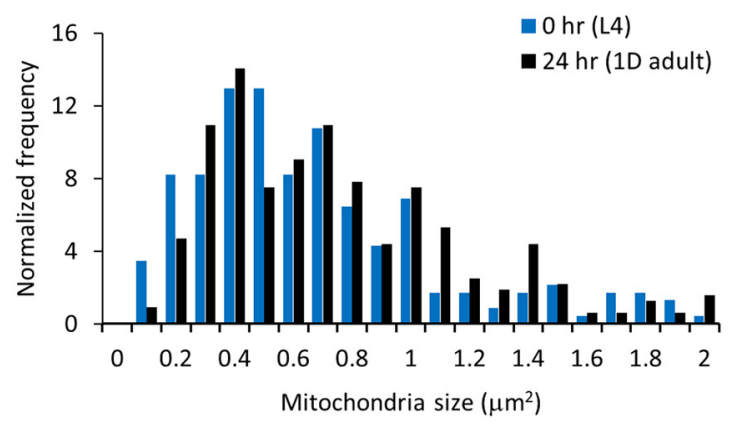

E

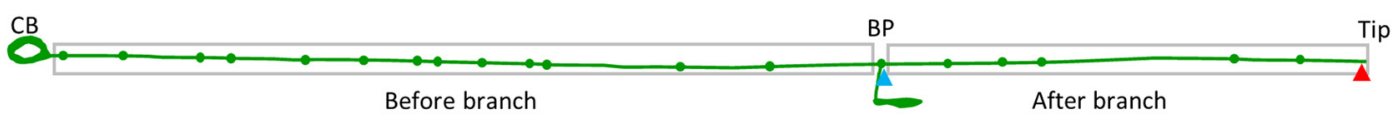

$\mathbf{F}$

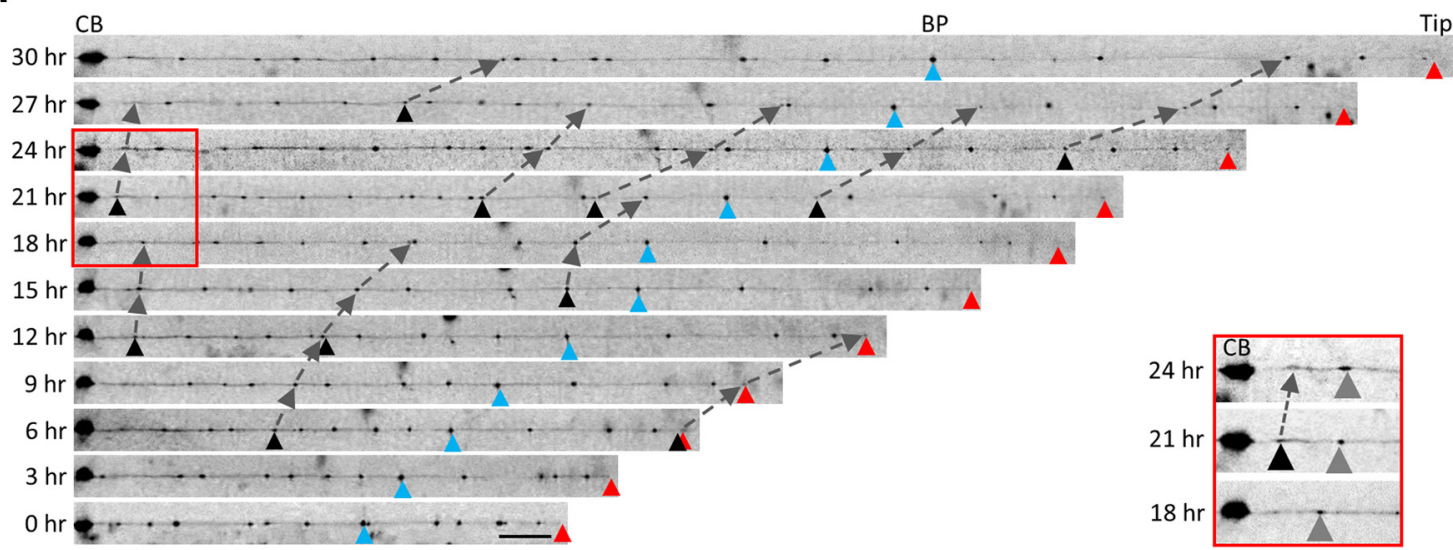

Figure 4. Total number of mitochondria increases linearly in TRNs in animals growing inside microfluidic devices. $\boldsymbol{A}$, The average number of mitochondria and the neuronal process length increases starting at $0 \mathrm{~h}(\mathrm{~L} 4)$ to $24 \mathrm{~h} \mathrm{(1D}$ adult) of imaging time inside the microfluidic device. $\boldsymbol{B}$, The ratio of the mitochondria number and the process length was unchanged over this $24 \mathrm{~h}$ imaging time period. The ratio is calculated for the values from the cell body (CB) to the BP and from the BP to the neuronal tip. Statistical significance was evaluated by paired sample $t$ test; ${ }^{*} p<0.05$, ${ }^{*} p<0.005$, and $\mathrm{ns}, p>0.05$ when comparing between the 0 - and 24-h imaging time point data. $\boldsymbol{C}$, Time course of the mitochondrial number and neuronal process length from eight animals imaged every $3 \mathrm{~h}$ in microfluidic devices beginning at L4 stage. Data represented as mean \pm SEM ( $n=8$ animals). $\boldsymbol{D}$, The relative frequency distribution of all mitochondria sizes at 0- and $24-h$ imaging time points ( $n=8$ animals). Statistical significance was evaluated by paired sample $t$ test $(p=0.92)$. $E$, A schematic of the TRN shows the neuronal process with the CB, the neuronal process end (Tip, red triangle), and the BP (blue triangle) as fiduciary markers. The BP is used to estimate the total mitochondria number and neuronal process length between the CB and the BP and between the BP and the end of the neuronal process. $\boldsymbol{F}$, The same animal was trapped inside the microfluidic device every $3 \mathrm{~h}$ and mitochondria imaged at high-resolution from the same TRN. Fluorescence images were straightened and raw images are displayed. The fiduciary markers of the CB, BP, and neuronal process end are used as references. New mitochondria added are labeled with black triangles and followed in two successive imaging time points and marked using dashed lines. Scale bar: $20 \mu \mathrm{m}$. The red box and the inset show a section of the neuronal process at 18-, 21-, and 24-h time points after start of imaging. A new mitochondrion (black triangle) is added in between the CB and a stationary mitochondrion (gray triangle). The distance between the $C B$ and the stationary mitochondria increases with time because of the growth of the neuronal process. Extended Data Figures 4-1, 4-2, 4-3 support this figure. 
mitochondria have low fluorescence intensity and appear randomly and intermittently along the TRN processes in our long-term images because of large time intervals between successive frames. The size of the stationary mitochondria in the neuronal process remains unchanged over longer time scales, i.e., $24 \mathrm{~h}$ (Fig. 4D). We consider a pair of mitochondria to be identical and stationary if the position of each mitochondrion from the cell body, BP, and tip of the neuron changes proportionately to the average growth rate of the neuron for that animal (typically $\sim 27 \mu \mathrm{m}$ per $3 \mathrm{~h}$ ). The neuronal processes of each individual TRN at multiple time points are aligned with each other from the cell body where the cell body, the BP, and the neuronal process tip act as reference points (Fig. 4F). With the growth of the neuronal process, the fiduciary markers (BP and tip) and all the mitochondria move further away from the cell body with time (Extended Data Fig. 4-1A). For every mitochondrion, the ratio of the new position to the old position was $1.1\left(L_{i}^{\prime} / L_{i}\right.$ where, $L_{i}^{\prime}$ and $L_{i}$ are the new and old position of the $i^{\text {th }}$ mitochondria with respect to the cell body), correlating with the $10 \%$ increase in the total neuronal process length over $3 \mathrm{~h}$. The uniform increase of the neuronal processes and the gradual shift in the stationary mitochondria positions are independent of the alignment of the neuronal process with respect to the cell body or the tip of the neuron (Extended Data Fig. 4-1B).

To identify a new mitochondrion addition, we identify a mitochondrion between a pair of preexisting stationary mitochondria with higher fluorescence than the background intensity from the neuronal process. The new mitochondrion must stay in the same location for at least two successive frames (i.e., $>6 \mathrm{~h}$ ). The addition of a new mitochondrion was identified using manual inspection of the relative mitochondrial position and geometrical parameters (size and integrated intensity) of the new mitochondrion after aligning the axons with respect to the fiduciary markers (Extended Data Fig. 4-1).

The number of mitochondria addition events was calculated for every equal third of the neuronal process, i.e., initial, middle, and end. The number of events in each third of the neuron was similar $33.3 \pm 3.24 \% \quad(n=29)$, $37.5 \pm 2.61 \% \quad(n=32)$, and $29.1 \pm 3.31 \% \quad(n=24)$ with $p \geq 0.07$ (Extended Data Fig. 4-2A; Table 2). We see that each new mitochondrion added has an approximate size of $0.47 \pm 0.07 \mu \mathrm{m}^{2}$ (mean \pm SEM) when first identified and grew to $0.68 \pm 0.11 \mu \mathrm{m}^{2}(n=4$ animals and $n=24$ new mitochondria addition events) after $3 \mathrm{~h}$. The gain in mitochondrial size can come from contributions from either the moving mitochondria or in situ biogenesis. Moving mitochondria along the neuronal processes are likely to initiate a new location and subsequently gain mitochondrial material by fusion-fission of moving mitochondria to form a larger stationary mitochondrion over time in the neuronal process. Using a long-term imaging platform, we were able to identify the addition of new mitochondria in an identified neuronal process.

\section{Increased intermitochondrial distance leads to the addition of a new mitochondrion in C. elegans TRNs}

The growth in the neuronal process increases the intermitochondrial distance between each adjacent pair of mitochondria. This growth of the neuron occurs along with new mitochondria addition such that density is maintained. To characterize changes in mitochondria density and identify the site of the new mitochondrion addition, we calculated the intermitochondrial distances between each adjacent pair of mitochondria (center-to-center distance along the neuronal process length) in all eight $L 4$ larval animals (Extended Data Fig. 4-2B). The average distance between adjacent mitochondria was 16.7 \pm 0.96 $\mu \mathrm{m}$ (mean $\pm \mathrm{SEM}$ ). To determine whether the distribution of mitochondria along the neuronal process was uniform, random, or clustered, we divided the neuronal processes from all eight animals into $20 \mu \mathrm{m}$ bins and counted the number of mitochondria per bin. The average number of mitochondria per bin $=1.0$ with a variance of $1.16 \pm 0.59$ (mean \pm variance, $n=101$ mitochondria), df $=4, \chi^{2}=$ 10.02 , and a confidence level $P_{(10.02,4)}=0.04$, suggesting a uniform distribution. Mitochondria can be added at different regions of the neuron altering intermitochondrial distances because of addition of new adjacent mitochondrial pairs (Extended Data Fig. 4-3). (1) Mitochondria can be added near the cell body; (2) at the end of the neuron; or (3) along the neuronal process when the intermitochondrial distance between adjacent mitochondria increases beyond a threshold. In either possibility (1) or (2) the intermitochondrial distance histograms will show two peaks, one at shorter and another at longer intermitochondrial distance intervals corresponding to additions in one region of the neuron and with increasing distance between other adjacent mitochondria pairs because of axon growth (Extended Data Fig. 4-3B,C). In the third possibility, as the mitochondria move apart because of increasing neuronal process length, the entire intermitochondrial distance distribution will shift to higher values (resulting in a low number of events for the short intermitochondrial distance values). In this case, a new mitochondrion will be added in between the old adjacent mitochondria pair and reducing the likelihood of longer intermitochondrial distance values. Hence, we expect to observe a small reduction in shorter intermitochondrial distances but a relatively unchanged longer intermitochondrial distance (Extended Data Fig. 4-3D).

Our data suggest that new mitochondrion addition occurs when the intermitochondrial distance between adjacent mitochondria increases beyond a threshold (orange; Fig. $5 A-D$ ). An adjacent pair of mitochondria moving apart by $25 \mu \mathrm{m}$ is still below the threshold and does not dock a new mitochondrion in between them (gray; Fig. $5 A, B$ ). To study mitochondrial addition in a growing neuronal process, we compared the intermitochondrial distances obtained from images at $0 \mathrm{~h}$ and $24 \mathrm{~h}$ of eight different animals at an early L4 and the 1D adult stages, respectively (Fig. 6A). After $24 \mathrm{~h}$, the population average of the intermitochondrial distances between adjacent mitochondria increased from $17 \pm 0.7$ to $19 \pm 0.6 \mu \mathrm{m}$ (mean \pm SEM, $n=8$ animals). To determine whether mitochondria addition on the neuronal process, continues to maintain a uniform distribution, we performed a $\chi^{2}$ analysis with all eight animals at the 24-h time point. We calculated the average number of mitochondria $(n=197)$ per bin $=1.0$ at $1.02 \pm 0.53$ (mean \pm variance), $\mathrm{df}=4, \chi^{2}=23.55$, and the 
A

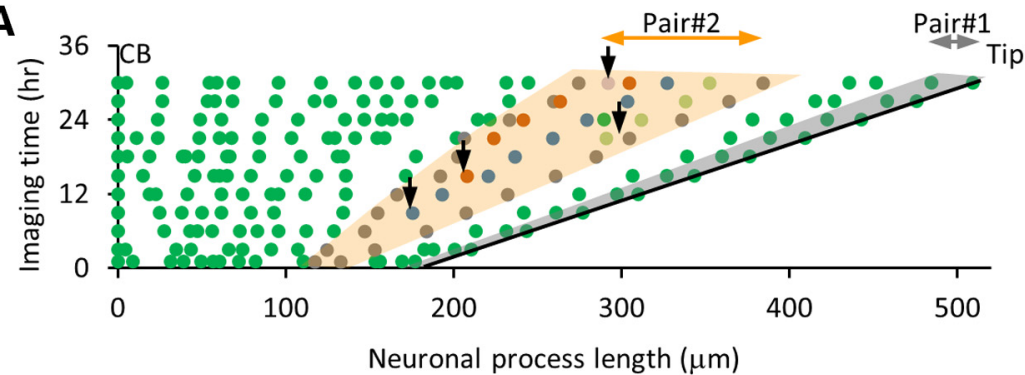

C

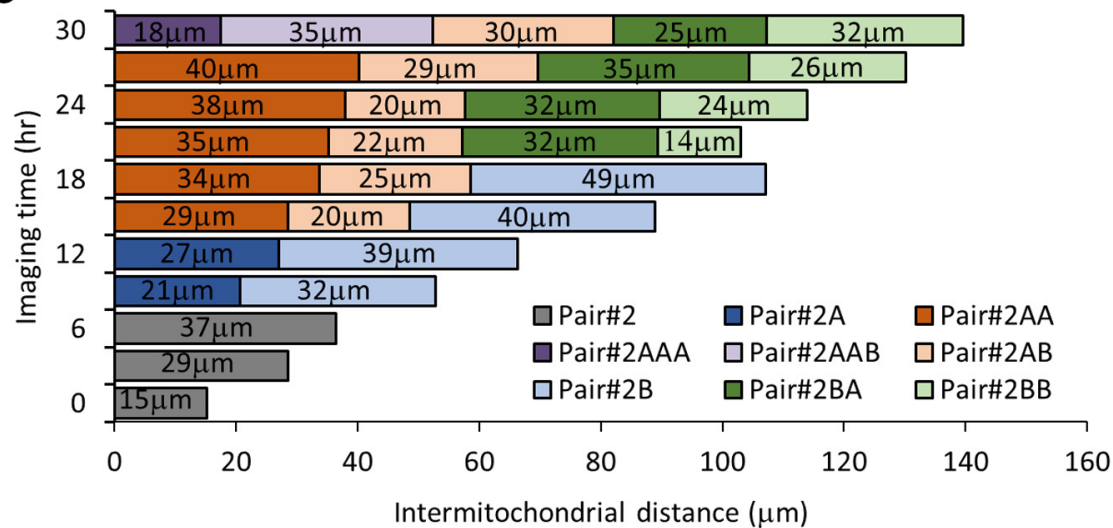

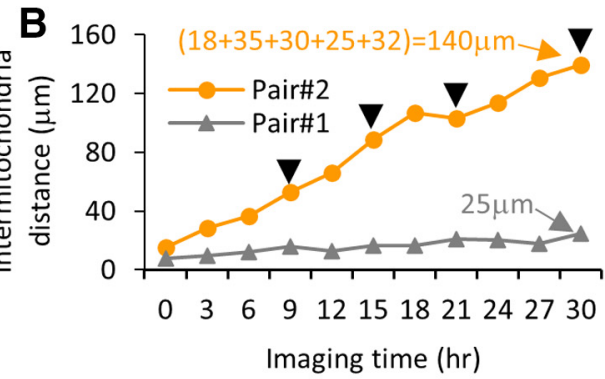

D Pair\#2AAA Pair\#2AAB

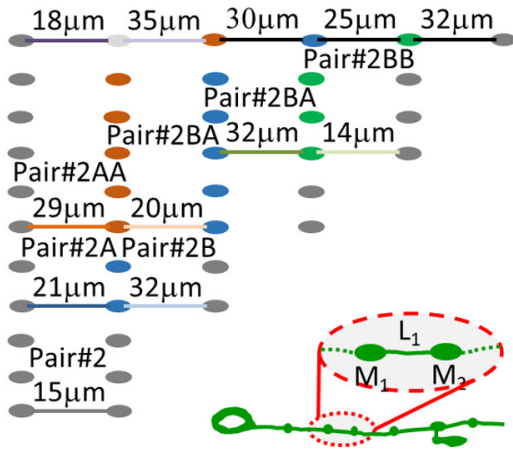

Total number of mitochondria in Pair\#2

Figure 5. Addition of new mitochondria during long-time imaging of the animal grown in the microfluidic device. A, Map of mitochondrial position from an individual C. elegans PLM neuron $36 \mathrm{~h}$ after hatching. Solid lines represent all two locations CB and Tip of the neuronal process. The shaded area in gray (pair \#1) is a region in the neuronal process where no mitochondrion is added between adjacent pairs in $30 \mathrm{~h}$ of imaging. The shaded area in orange (pair \#2) represents regions of the same neuronal process where four new mitochondria are identified between the adjacent pairs at different imaging times. The black arrows represent locations of all four new mitochondria. $\boldsymbol{B}$, Intermitochondrial intervals at different imaging times of two different pairs of stationary mitochondria (pairs \#1 and \#2) from an individual TRN process. The arrows indicate the time points when a new mitochondrion was added between the two adjacent mitochondria along the TRN process. The values indicate the total distance between parent adjacent pairs at the 30th hour of imaging, on adding all the intermediate intermitochondrial intervals formed because of new mitochondria additions. $\boldsymbol{C}$, The bar represents the intermitochondrial distances for pair \#2 measured from time-lapse imaging. Upon addition of a new mitochondrion, each pair of mitochondria is labeled as new and represented by two new colors (light and dark). The numbers on each bar indicate the intermitochondrial distances for every mitochondria pair. The shorter intermitochondrial distances in a few time points arise because of local compression/expansion of the neuronal process and are considered to be artifacts of imaging because of animal posture. $\boldsymbol{D}$, Schematic of the mitochondria lineage in pair \#2. The gray dots on the right represents the original adjacent mitochondria pair (not to the scale), while the new color dot indicates the location of the new mitochondrion added. Whenever a new mitochondrion is added, the intermitochondrial distances between new adjacent pairs are indicated by a line with a color similar to the color of the bars. The inset shows the schematic of a growing PLM neuron during the development of $C$. elegans. The cell body is on left and the neuronal process end is on right. The inset also shows two mitochondria $\left(M_{1}\right.$ and $\left.M_{2}\right)$ separated by length $L_{1}$.

confidence level $P_{(23.55,4)}=0.0001$ suggesting a uniform distribution. This analysis demonstrates that the neuronal processes continue to maintain a uniform distribution of mitochondria, even after multiple additions of new mitochondria. The addition of new mitochondria over $24 \mathrm{~h}$ causes a more skewed distribution of intermitochondrial distances as compared with the 0 -h time point (skewness and kurtosis measures increased from 0.81 and 0.34 at 0 $\mathrm{h}$ to 0.89 and 0.80 at $24 \mathrm{~h}$, respectively). The larger values for the skewness and kurtosis measured at $24 \mathrm{~h}$ indicate a strong right-tailed distribution, indicative of non-random mitochondrial positions as the neuronal process grows. Over the same $24 \mathrm{~h}$ period, the average values in the first two bins (\% of adjacent mitochondria separated by 3 and $6 \mu \mathrm{m})$ reduced from $7 \pm 1.03 \%$ and $9 \pm 1.27 \%$ to $3 \pm$ $1.17 \%$ and $7 \pm 1.01 \%(p=0.02$ and $p=0.17)$, respectively (Table 1). No other bin in the normalized intermitochondrial interval histogram was significantly different at the end of this imaging period. Although, lengthening of the neuronal process results in mitochondria moving apart (as indicated by the significant reduction in the shorter intermitochondrial intervals), the addition of new mitochondria ensures an insignificant increase in the larger intermitochondrial distance values. Although mitochondria undergo dynamic membrane fission/fusion and active transport (Sheng, 2014), their addition and positioning along the TRNs were surprisingly found to be regulated as reflected by the highly skewed distribution of the intermitochondrial distance values.

Adjacent intermitochondrial separation initiates the docking of a new mitochondrion forming two new adjacent independent pairs of mitochondria. One pair (pair \#1A) is with the old mitochondria close to the cell body and second pair (pair \#1B) is with the old mitochondria far from the cell body (Extended Data Fig. 6-1A,B). The intermitochondrial 


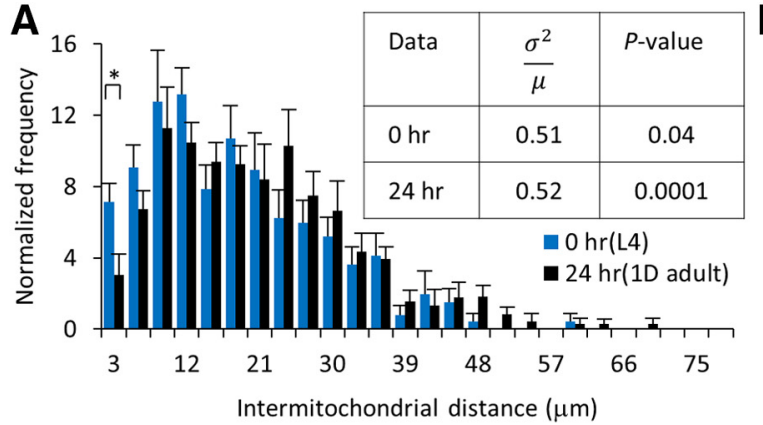

C
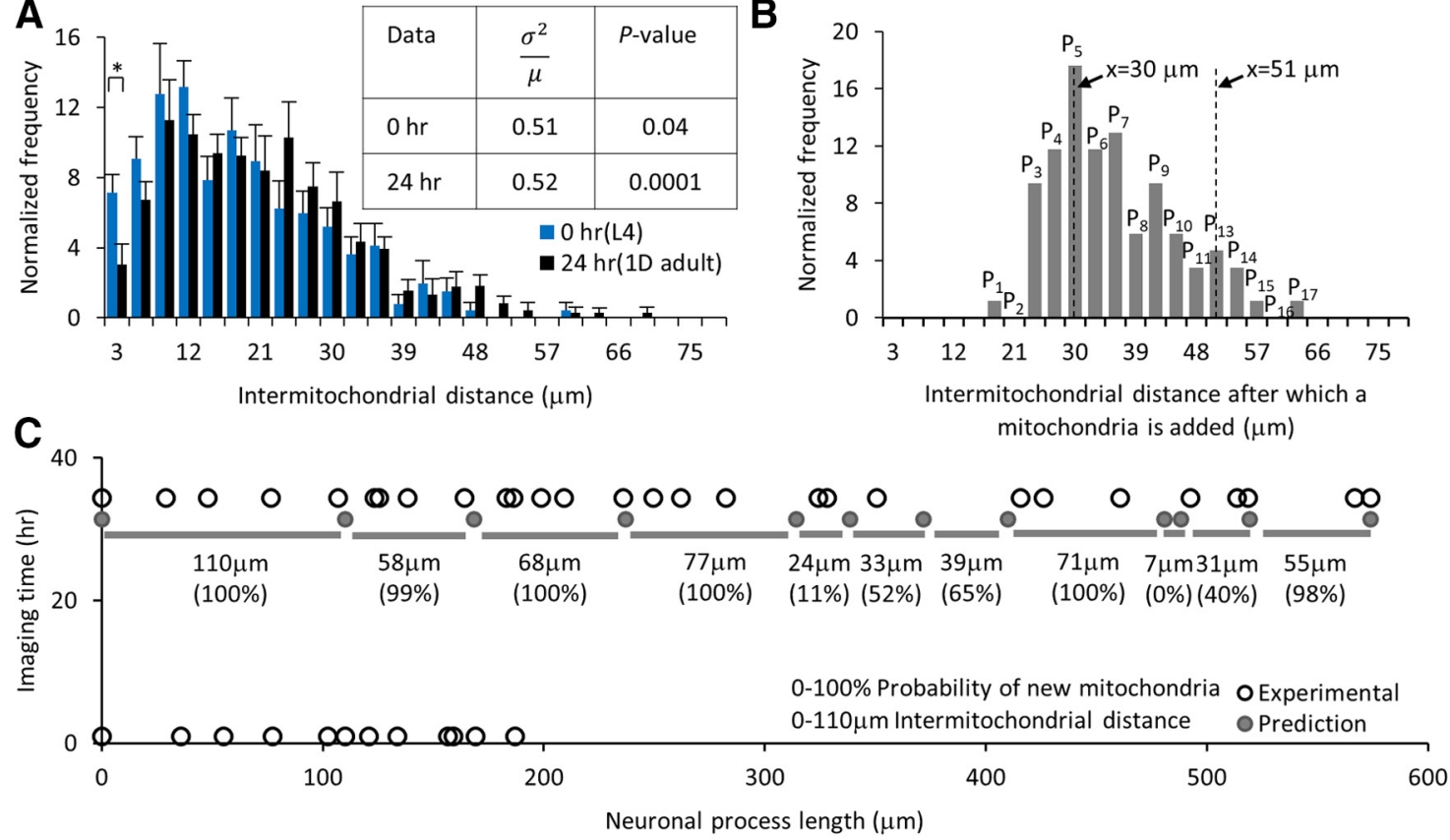

$$
\text { . }
$$

Figure 6. Experimental probability distribution can predict the addition of new mitochondria in a growing neuronal process. A, Normalized number of intermitochondrial distances at $0 \mathrm{~h}$ (L4) and $24 \mathrm{~h}$ (1D adult) after the start of imaging. Statistical significance was evaluated by two-sample $t$ test; ${ }^{*} p<0.05$ for the first two bars at bins 3 and $6 \mu \mathrm{m}$. The table represents the index of dispersion $\left(\frac{\sigma^{2}}{\mu}\right)$ and $p$ value obtained from the goodness of fit estimated using the $\chi^{2}$ analysis against a Poisson distribution for data from 0and 24-h imaging time points ( $n=8$ animals each). $\boldsymbol{B}$, Normalized number of mitochondria addition is calculated from experimental long-term images of individual TRNs ( $n=8$ animals started at the L4 stage, 85 new mitochondria additions). The probability of new mitochondria added for intermitochondrial distance $\mathrm{x}=\mathrm{L}$ is shown as $\mathrm{P}_{\mathrm{L}}$ and can be estimated by summing $\left(\sum_{i=1}^{L} P_{i}\right.$, where $\left.i \leq L\right)$. C, The predicted (dark circle) and experimental (open circle) location of mitochondria at 0 - and 33-h images of an individual animal. All the 12 positions at $t=33 \mathrm{~h}$ are predicted using the locations measured at $t=0 \mathrm{~h}$ and total neuronal process elongation values. The intermitochondrial distances (gray bars with the values) are used to predict the addition of new mitochondria between each pair at $33 \mathrm{~h}$. The probability values for finding new mitochondria within the predicted pairs are represented in percentages. Extended Data Figure 6-1 supports this figure.

distances between the old mitochondrial pair at the imaging time before a new mitochondrion is added show a distribution with an average interval of $34 \pm 1.0 \mu \mathrm{m}$ (mean \pm SEM, $n=85$ mitochondria addition events; Fig. 6B; Extended Data Fig. 6-1C). We identified all new mitochondria pairs to estimate the average distance for pair \#1A and pair \#1B as $17.1 \pm 0.8$ and $21 \pm 1.2 \mu \mathrm{m}$ (mean \pm SEM), respectively for all 85 events (Extended Data Fig. 6-1D). The new mitochondria addition events are uniformly distributed along the neuron (Extended Data Fig. 6-1E). Consistent with the third possibility (Extended Data Fig. 4-3D), we observed 85 events where two adjacent mitochondria had an intermitochondrial interval $>24 \mu \mathrm{m}$ when a new mitochondrion position appeared and remained for six or more hours. The addition of new mitochondria occurs uniformly throughout the neuron and maintains a similar average intermitochondrial distance with its adjacent mitochondria over a day of long-term imaging.

\section{Mitochondria positions can be estimated from the experimental probability distribution function}

Using all the individual events where a new mitochondrion was added, we calculated the experimental probability distribution of new addition events and predicted possible locations for the addition of new mitochondria using this probability distribution. The normalized plot of intermitochondrial distance (Fig. 6B) gives us the experimental probability distribution with a sum of all the values $\left(\sum P\right)=100 \%$. For any mitochondrial pair, we calculate the intermitochondrial distance $(x=L)$ and add normalized numbers of mitochondrial addition for all the intermitochondrial distances lower than the current value to assign the probability $\left(P_{L}\right)$ for the addition of a new mitochondrion between a given adjacent pair $\left(P_{L}=\sum_{i=1}^{L} P_{i}\right.$, where $i \leq L)$. To test our experimental probability distribution values, we imaged a neuronal process length $\left(\mathrm{L}_{0}=\right.$ $186.7 \mu \mathrm{m})$ with total mitochondria $\left(N_{0}=12\right)$ at time $t=0 \mathrm{~h}$ and predict that the hypothetical neuronal process grows to a length $\left(\mathrm{L}_{33}=573.7 \mu \mathrm{m}\right)$ at time $t=33 \mathrm{~h}$ after the start of imaging. This gave us 12 images that were collected every $3 \mathrm{~h}$ (Fig. 6C). For the "predicted" neuron, we calculate that the neuronal process elongates steadily with an experimental rate of $11 \mu \mathrm{m} / \mathrm{h}$, similar to the mean neuronal process elongation rate of $\sim 9 \mu \mathrm{m} / \mathrm{h}$. Using the above parameters, we estimate that the neuronal process (with an average intramitochondrial distance of $17 \pm 2.8 \mu \mathrm{m}$ at $t=0 \mathrm{~h}$ ) is predicted to grow $\sim 11 \mu \mathrm{m} / \mathrm{h}$ in $33 \mathrm{~h}$ leading to 

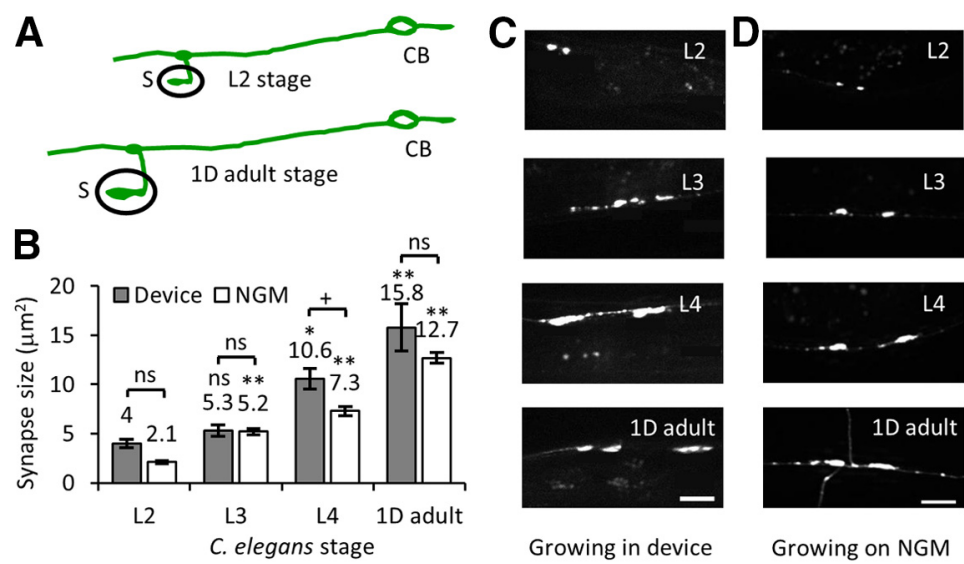

Figure 7. High-resolution imaging of GFP::RAB-3 in a developing C. elegans inside the microfluidic device. $\boldsymbol{A}$, Schematic of PLM neuron with its cell body (CB), its neuronal process, and its ventral synapse (S) at L2 larva and 1D adult stages. $\boldsymbol{B}$, The plot of synapse sizes at different developmental stages of $C$. elegans growing inside a microfluidic device and on NGM plates. Data represented as mean \pm SEM. Statistical significance was evaluated by one-way ANOVA with Bonferroni post hoc comparisons; ${ }^{*} p<$ 0.05 and ${ }^{* *} p<0.005$ when compared with data from L2 stage; $+p<0.05$ and $++p<0.005$, ns, $p<0.05$ when compared between animals grown in the device and on NGM solid media; nonsignificant comparisons are not indicated. The number of synapses analyzed from animals grown on NGM solid media is $n \geq 37$ and in devices are $n=18$ (L2), 16 (L3), 14 (L4), and 8 (1D adult). C, $\boldsymbol{D}$, Images of PLM synapses at L2, L3, L4, and 1D adult stages of development grown in the microfluidic device (C) and on NGM solid media plates and imaged on agar pads $(\boldsymbol{D})$. Scale bar: $10 \mu \mathrm{m}$.

an average intermitochondrial interval of $52 \pm 8.7 \mu \mathrm{m}$, without the addition of new mitochondria (Fig. 6C). When the separation between adjacent mitochondrial pairs increases beyond $24 \mu \mathrm{m}$, we predict the addition of a new mitochondrion (Fig. 6B). This prediction is supported by our experimental observations (Fig. 6C). The addition of a new mitochondrion between two stationary mitochondria, as the neuronal process expands with the growth of an animal, is controlled by the rate of neuronal elongation, a gradual increase in the intermitochondrial distances beyond the $24 \mu \mathrm{m}$ threshold, and predicted by the experimental probability distribution.

This technology allows us, for the first time, to track individual neurons in vivo to assess dynamics that occur over slow timescales such as intracellular organelle positioning across development. Using our long-term imaging device, we were able to extract the experimental probability distribution for organelle addition along the axon and use the values to predict the location of a new mitochondrion addition between a pair of adjacent preexisting mitochondria in a growing neuron. Multiple factors could determine the addition of new mitochondria such as the ratio of molecular motors (Sure et al., 2018; Chen et al., 2021), local cytoskeleton (Sood et al., 2018), local calcium concentration (Wang and Schwarz, 2009b), new docking sites (Cai and Sheng, 2009), and/or reactive oxygen species (Debattisti et al., 2017; Liao et al., 2017).

\section{Our long-term device can be used to study synaptic development in an individual animal}

We wanted to test whether our device could be used to image other developmental processes to examine its general utility for other types of biological processes. To perform high-resolution time-lapse imaging of subcellular organelle accumulation events in C. elegans across developmental stages, we captured fluorescence images of GFP::RAB-3 accumulation at the ventral PLM synapses from the same individual js/s821 animals (Nonet, 1999; Mahoney et al., 2006; Kumar et al., 2010). We immobilized each animal using our imaging platform under 14 psi pressure inside the flow channel. Slow progressive immobilization provides a suitable lateral orientation of $C$. elegans and the best view of the PLM synapses (Fig. 7). We immobilized the same animal in a lateral orientation to image both PLM synapses at L2 (16 h), L3 (22 h), L4 (38 h), and 1D adult $(68 \mathrm{~h})$ stages from the time the eggs hatched. High-resolution time-lapse images were analyzed for synapse sizes to estimate synapse growth at different developmental stages. The amount of GFP::RAB-3 accumulation shows a gradual increase similar to the prior average signal measured from multiple populations of $C$. elegans animals immobilized inside the microfluidic device at different developmental stages (Mondal et al., 2011). This increase is thought to arise from the gradual increase in the net anterograde flux of SVs (Mondal et al., 2011). Our imaging platform captured a robust increase in the accumulation of GFP::RAB-3 at the synapses of the same animal over multiple developmental stages.

\section{Discussion}

C. elegans TRNs grow from $\sim 100 \mu \mathrm{m}$ at the $\mathrm{L} 1$ larval stage to $500 \mu \mathrm{m}$ in 1D adult stage. During this growth, $\sim 20$ new mitochondria are added to maintain a constant mitochondrial density of approximately five mitochondria/ $100 \mu \mathrm{m}$ along the neuronal process (Fig. 1D,E). The density measured in adult animals grown in our microfluidic device is very similar to that previously reported in young adult animals (Morsci et al., 2016; Sure et al., 2018). Conventional anesthetic assays can have detrimental effects on animal physiology, i.e., it can alter muscle 
Table 1: Statistical analysis for different figures

\begin{tabular}{ll}
\hline Figure & Normality \\
\hline Fig. $1 E$ & Yes
\end{tabular}

Test used

\begin{tabular}{ll}
$p$ value & Power \\
\hline 1.000 & 0.772 \\
0.091 & \\
0.016 & \\
0.689 &
\end{tabular}

Fig. $2 H$

Yes

Two-sample $t$ test

0.075

0.391

0.129

$<0.005$

1.000

0.418

0.120

0.027

0.645

0.003

0.947

Fig. 2 I

Yes

Two-sample $t$ test

0.021

0.658

0.012

0.779

0.083

0.414

0.012

0.778

0.815

0.143

0.303

Fig. $3 D$

No

Two-sample $t$ test

0.06

0.433

Fig. $3 E$

No

Two-sample $t$ test

0.06

0.403

Fig. $3 F$

No

Two-sample $t$ test

0.01

0.928

0.14

0.348

Fig. $3 G$

Yes

One-way ANOVA with Bonferroni post hoc

0.20

0.239

0.10

0.355

Fig. 3l

No

Two-sample $t$ test

0.004

0.0917

0.01

0.07

0.199

0.02

0.466

0.03

0.238

$<0.005$

0.363

$<0.005$

0.482

0.04

0.271

Fig. 3J

No

Two-sample $t$ test

$<0.005$

0.509

$<0.005$

0.630

$<0.005$

0.552

0.002

0.457

$>0.05$

0.145

$>0.05$

0.086

Fig. 3K

Yes

Two-sample $t$ test

0.15

0.290

0.45

0.113

Fig. $3 L$

Yes

Two-sample $t$ test

0.30

0.174

0.01

0.683

Fig. $3 M$

Yes

Two-sample $t$ test

0.07

0.447

$>0.05$

0.380

Extended Data Fig. 3-2H

Yes

Two-sample $t$ test

0.50

0.47

$4 A$

Yes

Two-sample $t$ test

$<0.005$

0.999

$<0.005$ 1

Fig. $4 B$

Yes

Two-sample $t$ test

0.96

0.050

0.13

0.321

(Continued) 
Table 1: Continued

\begin{tabular}{|c|c|c|c|c|}
\hline Figure & Normality & Test used & $p$ value & Power \\
\hline Fig. $4 D$ & Yes & Two-sample $t$ test & 0.92 & 0.051 \\
\hline Extended Data Fig. 4-2A & Yes & One-way ANOVA with post hoc Tukey & $\begin{array}{l}0.602 \\
0.606\end{array}$ & 0.346 \\
\hline $6 A$ & Yes & Two-sample $t$ test & $\begin{array}{l}0.02 \\
0.17\end{array}$ & $\begin{array}{l}0.692 \\
0.268\end{array}$ \\
\hline $7 B$ & Yes & One-way ANOVA with Bonferroni post hoc & $\begin{array}{l}1.0 \\
<0.005 \\
<0.005 \\
<0.005 \\
<0.005 \\
<0.005 \\
0.74 \\
1.0 \\
0.01 \\
0.18\end{array}$ & 1 \\
\hline
\end{tabular}

tone consequently altering body length, and cannot easily be used for long-term imaging of the same animal. Anesthetic-free immobilization using a microfluidic device shows robust vesicle transport (Mondal et al., 2011), fast axonal regeneration rates (Guo et al., 2008), and quick behavioral recovery (Dong et al., 2018; Manjarrez and Mailler, 2020). In agreement with our previous vesicle transport studies (Mondal et al., 2011), we found robust mitochondrial transport in the device immobilized animals. To follow slow mitochondria addition during neuronal process growth, we developed a microfluidic device that can be used to grow $C$. elegans throughout its development without significant adverse effects on its growth and health (Fig. $2 A-F$ ). Its easy operation enables continuous imaging of the same animal inside the microfluidic environment to quantify in vivo subcellular process in the neuron throughout development. Mitochondria in the TRNs were found to show lower flux in the microfluidic device compared with anesthetic immobilization (Fig. 3D). These observations are similar to the lower flux of SVs when immobilized in microfluidic devices compared with anesthetic immobilization (Mondal et al., 2011). Using long-term imaging over a period of $36 \mathrm{~h}$, we identified mitochondria addition and changes in intermitochondrial distances in C. elegans TRNs during its elongation after synapse formation (Fig. 4F). Using our microfluidic device platform, one can monitor slow processes such as the addition of mitochondria. Slow entry and exit and limited turn-over of mitochondria identified in our imaging platform suggest that the neuron retains positions of old mitochondria and adds mitochondria at new locations between preexisting mitochondria along the growing process during development (Fig. 4F).

New mitochondrion addition during growth and axon stretching has been shown to occur between adjacent pairs of stationary mitochondria (Miller and Sheetz, 2004; Lamoureux et al., 2010; Misko et al., 2012; Matsumoto et al., 2020). Our data are consistent with prior observations where we see a uniform distribution of mitochondria, proportionate growth of the neuron, and addition of mitochondria occurring anywhere along the process between two stationary mitochondria (Fig. 5). This mitochondrion addition occurs anywhere along the neuronal process whenever the average separation between two nearest stationary mitochondrial neighbors is at least $24 \mu \mathrm{m}$ (Fig. $6 B)$, suggesting the presence of a signal that detects intermitochondrial distances.

We have identified the experimental probability distribution for the addition of a new mitochondrion in a growing neuronal process in vivo over a $24 \mathrm{~h}$ period. The distribution for the intermitochondrial distances becomes more skewed and the mean intermitochondrial distance

Table 2: Table representing the transport parameters and the turnover of mitochondria at stationary mitochondria from L4 animals anesthetized using the microfluidic device and $3 \mathrm{mM}$ levamisole

\begin{tabular}{|c|c|c|c|c|}
\hline & \multicolumn{2}{|c|}{ Device } & \multicolumn{2}{|c|}{$3 \mathrm{mM}$ levamisole } \\
\hline & Init PLM & Mid PLM & Init PLM & Mid PLM \\
\hline Retrogradely moving cross & $0 \%$ & $0 \%$ & $17 \%$ & $18 \%$ \\
\hline Retrogradely moving contributes to recovery & $0 \%$ & $17 \%$ & $21 \%$ & $21 \%$ \\
\hline Total movies/total events analyzed & $8 / 6$ & $12 / 6$ & $29 / 88$ & $37 / 109$ \\
\hline Anterograde velocity (Device) & \multicolumn{2}{|c|}{$0.40 \pm 0.042(n=47)$} & \multicolumn{2}{|c|}{$0.29 \pm 0.011(n=336)$} \\
\hline
\end{tabular}


increases with the development of the animal (Fig. 6A). Although new mitochondria are added over the 24-h time, the index of dispersion indicates that the distribution remains uniform (Fig. 6A table) strengthening the argument that addition is not a random process. As development and growth occur, the number of closely spaced mitochondria is reduced, decreasing the fraction of mitochondria with lower separation between them. During neuronal process elongation, the new mitochondria added are small in size and have low integrated fluorescence intensity, they increase in size and intensity over time (Extended Data Fig. 4-1). There are two possible mechanisms for the addition of a new mitochondrion that remains a subject for further studies: (1) a small mitochondrion enters from the cell body, moves along the neuronal process, identifies a large intermitochondrial separation, and anchors within the "mitochondria-in-demand" region; and (2) a local mitochondrion fission event causes a new small mitochondrion to emerge from a nearby large mitochondrion, moves a shorter distance, and anchors within the mitochondria-in-demand region. However, we have not observed a large stationary mitochondrion show such fission in our imaging. All events appear to be initiated by moving mitochondria undergoing fusion or fission with a preexisting stationary mitochondrion.

Stationary mitochondria when photobleached using a high-power laser, lose nearly $96.5 \%$ of the fluorescence intensity instantaneously (in $0.33 \mathrm{~s}$ when captured at $3 \mathrm{fps}$ rate; Extended Data Fig. 3-2B). We think our bleaching protocol is unlikely to damage mitochondria sufficiently to make them generally permeable to cytoplasmic GFP or GFP coming from another mitochondrion that crosses this bleached stationary mitochondrion. We observe several examples where rapidly moving mitochondria cross photobleached mitochondrion locations without any significant change in fluorescence intensity of the moving or the photobleached mitochondria (Extended Data Fig. 3$2 D-F$ ). Only a subset of moving mitochondria interacted with a photobleached mitochondrion.

Halting of a mitochondrion at the new mitochondria-indemand region is likely to be facilitated by several factors such as motors, local calcium levels, local ATP concentrations, cytoskeleton architectures, etc. Thus, the addition of new mitochondria likely arises from the stalling of moving mitochondria at a location, between a pair of previously docked mitochondria, with a probability that increases with the increasing separation between two adjacent stationary mitochondria. The stalled mitochondria appear to enlarge in size potentially through fusion with material from moving mitochondria that go past it. We believe that time-lapse imaging of adjacent mitochondrial pairs $>24 \mu \mathrm{m}$ apart, identified from our longterm time-lapse imaging platform, could shed light on mechanisms of mitochondrial addition. Time-lapse imaging of fission mutants and animals with transport defects can also help delineate the underlying processes and molecular players important in new mitochondria addition events. The long-term mitochondria dynamics studies in $C$. elegans models using a microfluidic platform not only offers a robust assay to study organelle transport in vivo, but it provides an opportunity to move beyond simple observations obtained from in vitro assays to discover genes that can regulate the positioning of mitochondria in neurons.

Our device can be used at different time intervals. For studies that require more frequent imaging of the same animal, we immobilize the same animal every hour for 12 h (Extended Data Fig. 3-1). A 1 h more frequent immobilization showed greater but statistically insignificant photobleaching when imaged at $12 \mathrm{~h}$ time point (Extended Data Fig. 3-1C). Intermittent long-term imaging separated by $\sim 6 \mathrm{~h}$ for $52 \mathrm{~h}$ shows a steady increase in synaptic accumulation of GFP::RAB-3 when measured from an individual animal grown inside the microfluidic device. The amount of accumulation was slightly higher in devicegrown animals as compared with animals grown on NGM plates (Fig. 7; Mondal et al., 2011). This increase compared with animals grown on NGM plates may arise from additional mechanical stimulation provided by the deflected membrane pressing against the animal. Despite these caveats, our microfluidic platform can be used for a variety of studies to assess long-term cellular or subcellular dynamics in $C$. elegans. Similar technologies can be used for other model organisms by modifying the channel geometries (Mondal et al., 2011, 2012).

\section{References}

Atakan HB, Xiang R, Cornaglia M, Mouchiroud L, Katsyuba E, Auwerx J, Gijs MAM (2019) Automated platform for long-term culture and high-content phenotyping of single $C$. elegans worms. Sci Rep 9:14340.

Awasthi A, Modi S, Hegde S, Chatterjee A, Mondal S, Romero E, Sure GR, Koushika SP (2020) Regulated distribution of mitochondria in touch receptor neurons of $C$. elegans influences touch response. bioRxiv. doi: 10.1101/2020.07.26.221523.

Brenner S (1974) The genetics of Caenorhabditis elegans. Genetics 77:71-94.

Cáceres IdeC, Valmas N, Hilliard MA, Lu H (2012) Laterally orienting C. elegans using geometry at microscale for high-throughput visual screens in neurodegeneration and neuronal development studies. PLoS One 7:e35037.

Cai Q, Sheng ZH (2009) Mitochondrial transport and docking in axons. Exp Neurol 218:257-267.

Cai Q, Tammineni P (2017) Mitochondrial aspects of synaptic dysfunction in Alzheimer's disease. J Alzheimers Dis 57:1087-1103.

Chalasani SH, Chronis N, Tsunozaki M, Gray JM, Ramot D, Goodman MB, Bargmann Cl (2007) Dissecting a circuit for olfactory behaviour in Caenorhabditis elegans. Nature 450:63-70.

Chen Y, Sheng ZH (2013) Kinesin-1-syntaphilin coupling mediates activity-dependent regulation of axonal mitochondrial transport. $J$ Cell Biol 202:351-364.

Chen YC, Huang HR, Hsu CH, Ou CY (2021) CRMP/UNC-33 organizes microtubule bundles for KIF5-mediated mitochondrial distribution to axon. PLoS Genet 17:e1009360.

Chiu SY (2011) Matching mitochondria to metabolic needs at nodes of Ranvier. Neuroscientist 17:343-350.

Chung K, Crane MM, Lu H (2008) Automated on-chip rapid microscopy, phenotyping and sorting of C. elegans. Nat Methods 5:637643.

Debattisti V, Gerencser AA, Saotome M, Das S, Hajnoczky G (2017) ROS control mitochondrial motility through p38 and the motor adaptor Miro/Trak. Cell Rep 21:1667-1680. 
Dong L, Cornaglia M, Krishnamani G, Zhang J, Mouchiroud L, Lehnert T, Auwerx J, Gijs MAM (2018) Reversible and long-term immobilization in a hydrogel-microbead matrix for high-resolution imaging of Caenorhabditis elegans and other small organisms. PLoS One 13:e0193989.

Fatouros C, Pir Gj, Biernat J, Koushika SP, Mandelkow E, Mandelkow EM, Schmidt E, Baumeister R (2012) Inhibition of tau aggregation in a novel Caenorhabditis elegans model of tauopathy mitigates proteotoxicity. Hum Mol Genet 21:3587-3603.

Freedman D, Diaconis $P$ (1981) On the histogram as a density estimator: L2 theory. Z Wahrscheinlichkeitstheorie verw Gebiete 57:453-476.

Gokce SK, Hegarty EM, Mondal S, Zhao P, Ghorashian N, Hilliard Ma, Ben-Yakar A (2017) A multi-trap microfluidic chip enabling longitudinal studies of nerve regeneration in Caenorhabditis elegans. Sci Rep 7:9837.

Guo SX, Bourgeois F, Chokshi T, Durr NJ, Hilliard MA, Chronis N, Ben-Yakar A (2008) Femtosecond laser nanoaxotomy lab-on-achip for in vivo nerve regeneration studies. Nat Methods 5:531533.

Hancock WO (2014) Bidirectional cargo transport: moving beyond tug of war. Nat Rev Mol Cell Biol 15:615-628.

Hill S, Van Remmen H (2014) Mitochondrial stress signaling in longevity: a new role for mitochondrial function in aging. Redox Biol 2:936-944.

Hobert O (2010) Neurogenesis in the nematode Caenorhabditis elegans. Advance online publication. Retrieved Oct 4, 2010. doi: 10.1895/wormbook.1.12.2.

Hulme SE, Shevkoplyas SS, Mcguigan AP, Apfeld J, Fontana W, Whitesides GM (2010) Lifespan-on-a-chip: microfluidic chambers for performing lifelong observation of $C$. elegans. Lab Chip 10:589-597.

Jovaisaite V, Mouchiroud L, Auwerx J (2014) The mitochondrial unfolded protein response, a conserved stress response pathway with implications in health and disease. J Exp Biol 217:137-143.

Kang JS, Tian JH, Pan PY, Zald P, Li C, Deng C, Sheng ZH (2008) Docking of axonal mitochondria by syntaphilin controls their mobility and affects short-term facilitation. Cell 132:137-148.

Keil W, Kutscher LM, Shaham S, Siggia ED (2017) Long-term highresolution imaging of developing $C$. elegans larvae with microfluidics. Dev Cell 40:202-214.

Krajniak J, Lu H (2010) Long-term high-resolution imaging and culture of $C$. elegans in chip-gel hybrid microfluidic device for developmental studies. Lab Chip 10:1862-1868.

Krajniak J, Hao Y, Mak HY, Lu H (2013) C.L.I.P.-continuous live imaging platform for direct observation of $C$. elegans physiological processes. Lab Chip 13:2963-2971.

Kumar J, Choudhary BC, Metpally R, Zheng Q, Nonet ML, Ramanathan S, Klopfenstein DR, Koushika SP (2010) The Caenorhabditis elegans Kinesin-3 motor UNC-104/KIF1A is degraded upon loss of specific binding to cargo. PLoS Genet 6: e1001200.

Labbadia J, Brielmann RM, Neto MF, Lin YF, Haynes CM, Morimoto $\mathrm{RI}$ (2017) Mitochondrial stress restores the heat shock response and prevents proteostasis collapse during aging. Cell Rep 21:1481-1494.

Lamoureux P, Heidemann SR, Martzke NR, Miller KE (2010) Growth and elongation within and along the axon. Dev Neurobiol 70:135149.

Lee H, Kim SA, Coakley S, Mugno P, Hammarlund M, Hilliard MA, Lu H (2014) A multi-channel device for high-density target-selective stimulation and long-term monitoring of cells and subcellular features in C. elegans. Lab Chip 14:4513-4522.

Liao PC, Tandarich LC, Hollenbeck PJ (2017) ROS regulation of axonal mitochondrial transport is mediated by $\mathrm{Ca}^{2+}$ and $\mathrm{JNK}$ in Drosophila. PLoS One 12:e0178105.

Liu XA, Rizzo V, Puthanveettil SV (2012) Pathologies of axonal transport in neurodegenerative diseases. Transl Neurosci 3:355-372.

Mahoney TR, Liu Q, Itoh T, Luo S, Hadwiger G, Vincent R, Wang ZW, Fukuda M, Nonet ML (2006) Regulation of synaptic transmission by RAB-3 and RAB-27 in Caenorhabditis elegans. Mol Biol Cell 17:2617-2625.

Manjarrez JR, Mailler R (2020) Stress and timing associated with Caenorhabditis elegans immobilization methods. Heliyon 6: e04263.

Matsumoto N, Hori I, Murase T, Tsuji T, Miyake S, Inatani M, Konishi $Y(2020)$ Intermitochondrial signaling regulates the uniform distribution of stationary mitochondria in axons. bioRxiv. doi: 10.1101/ 2020.07.31.230250.

Melentijevic I, Toth ML, Arnold ML, Guasp RJ, Harinath G, Nguyen KC, Taub D, Parker JA, Neri C, Gabel CV, Hall DH, Driscoll M (2017) C. elegans neurons jettison protein aggregates and mitochondria under neurotoxic stress. Nature 542:367-371.

Millecamps S, Julien JP (2013) Axonal transport deficits and neurodegenerative diseases. Nat Rev Neurosci 14:161-176.

Miller KE, Sheetz MP (2004) Axonal mitochondrial transport and potential are correlated. J Cell Sci 117:2791-2804.

Misgeld T, Schwarz TL (2017) Mitostasis in neurons: maintaining mitochondria in an extended cellular architecture. Neuron 96:651666.

Misko AL, Sasaki Y, Tuck E, Milbrandt J, Baloh RH (2012) Mitofusin2 mutations disrupt axonal mitochondrial positioning and promote axon degeneration. J Neurosci 32:4145-4155.

Mondal S, Koushika SP (2014) Microfluidic devices for imaging trafficking events in vivo using genetic model organisms. Methods Mol Biol 1174:375-396.

Mondal S, Ahlawat S, Rau K, Venkataraman V, Koushika SP (2011) Imaging in vivo neuronal transport in genetic model organisms using microfluidic devices. Traffic 12:372-385.

Mondal S, Ahlawat S, Koushika SP (2012) Simple microfluidic devices for in vivo imaging of $C$. elegans, Drosophila and zebrafish. J Vis Exp. Advance online publication. Retrieved Sep 30, 2012. doi: $10.3791 / 3780$.

Mondal S, Hegarty E, Martin C, Gokce SK, Ghorashian N, Ben-Yakar A (2016) Large-scale microfluidics providing high-resolution and high-throughput screening of Caenorhabditis elegans poly-glutamine aggregation model. Nat Commun 7:13023.

Mondal S, Hegarty E, Sahn JJ, Scott LL, Gokce SK, Martin C, Ghorashian N, Satarasinghe PN, lyer S, Sae-Lee W, Hodges TR, Pierce JT, Martin SF, Ben-Yakar A (2018) High-content microfluidic screening platform used to identify sigma2R/Tmem97 binding ligands that reduce age-dependent neurodegeneration in $C$. elegans SC_APP model. ACS Chem Neurosci 9:1014-1026.

Morris RL, Hollenbeck PJ (1993) The regulation of bidirectional mitochondrial transport is coordinated with axonal outgrowth. J Cell Sci 104:917-927.

Morsci NS, Hall DH, Driscoll M, Sheng ZH (2016) Age-related phasic patterns of mitochondrial maintenance in adult Caenorhabditis elegans neurons. J Neurosci 36:1373-1385.

Nonet ML (1999) Visualization of synaptic specializations in live C. elegans with synaptic vesicle protein-GFP fusions. J Neurosci Methods 89:33-40.

O'Toole M, Latham R, Baqri RM, Miller KE (2008) Modeling mitochondrial dynamics during in vivo axonal elongation. $J$ Theor Biol 255:369-377.

Obashi K, Okabe S (2013) Regulation of mitochondrial dynamics and distribution by synapse position and neuronal activity in the axon. Eur J Neurosci 38:2350-2363.

Ohno N, Kidd GJ, Mahad D, Kiryu-Seo S, Avishai A, Komuro H, Trapp BD (2011) Myelination and axonal electrical activity modulate the distribution and motility of mitochondria at CNS nodes of Ranvier. J Neurosci 31:7249-7258.

Petzold BC, Park SJ, Ponce P, Roozeboom C, Powell C, Goodman MB, Pruitt BL (2011) Caenorhabditis elegans body mechanics are regulated by body wall muscle tone. Biophys J 100:1977-1985.

Rahman M, Hewitt JE, Van-Bussel F, Edwards H, Blawzdziewicz J, Szewczyk NJ, Driscoll M, Vanapalli SA (2018) NemaFlex: a microfluidics-based technology for standardized measurement of muscular strength of C. elegans. Lab Chip 18:2187-2201. 
Samara C, Rohde CB, Gilleland CL, Norton S, Haggarty SJ, Yanik MF (2010) Large-scale in vivo femtosecond laser neurosurgery screen reveals small-molecule enhancer of regeneration. Proc Natl Acad Sci USA 107:18342-18347.

Schwarz TL (2013) Mitochondrial trafficking in neurons. Cold Spring Harb Perspect Biol 5:a011304.

Seervi M, Xue D (2015) Mitochondrial cell death pathways in Caenorhabditis elegans. Curr Top Dev Biol 114:43-65.

Sheng ZH (2014) Mitochondrial trafficking and anchoring in neurons: new insight and implications. J Cell Biol 204:1087-1098.

Sheng ZH, Cai Q (2012) Mitochondrial transport in neurons: impact on synaptic homeostasis and neurodegeneration. Nat Rev Neurosci 13:77-93.

Sison M, Chakrabortty S, Extermann J, Nahas A, James Marchand P, Lopez A, Weil T, Lasser T (2017) 3D time-lapse imaging and quantification of mitochondrial dynamics. Sci Rep 7:43275.

Smith GM, Gallo G (2018) The role of mitochondria in axon development and regeneration. Dev Neurobiol 78:221-237.

Smith CJ, Watson JD, Spencer WC, O'Brien T, Cha B, Albeg A, Treinin M, Miller DM 3rd (2010) Time-lapse imaging and cell-specific expression profiling reveal dynamic branching and molecular determinants of a multi-dendritic nociceptor in C. elegans. Dev Biol 345:18-33.

Solis GM, Petrascheck M (2011) Measuring Caenorhabditis elegans life span in 96 well microtiter plates. J Vis $\operatorname{Exp}(49): 2496$.

Sood P, Murthy K, Kumar V, Nonet ML, Menon GI, Koushika SP (2018) Cargo crowding at actin-rich regions along axons causes local traffic jams. Traffic 19:166-181.
Sun N, Youle RJ, Finkel T (2016) The mitochondrial basis of aging. Mol Cell 61:654-666.

Sun T, Qiao H, Pan PY, Chen Y, Sheng ZH (2013) Motile axonal mitochondria contribute to the variability of presynaptic strength. Cell Rep 4:413-419.

Sure GR, Chatterjee A, Mishra N, Sabharwal V, Devireddy S, Awasthi A, Mohan S, Koushika SP (2018) UNC-16/JIP3 and UNC-76/FEZ1 limit the density of mitochondria in $C$. elegans neurons by maintaining the balance of anterograde and retrograde mitochondrial transport. Sci Rep 8:8938.

Varier S, Kaiser M (2011) Neural development features: spatio-temporal development of the Caenorhabditis elegans neuronal network. PLoS Comput Biol 7:e1001044.

Wang X, Schwarz TL (2009a) Imaging axonal transport of mitochondria. Methods Enzymol 457:319-333.

Wang X, Schwarz TL (2009b) The mechanism of Ca2+-dependent regulation of kinesin-mediated mitochondrial motility. Cell 136:163-174.

Welte MA (2004) Bidirectional transport along microtubules. Curr Biol 14:R525-R537.

Whitesides GM, Ostuni E, Takayama S, Jiang X, Ingber DE (2001) Soft lithography in biology and biochemistry. Annu Rev Biomed Eng 3:335-373.

Xian B, Shen J, Chen W, Sun N, Qiao N, Jiang D, Yu T, Men Y, Han Z, Pang Y, Kaeberlein M, Huang Y, Han JD (2013) WormFarm: a quantitative control and measurement device toward automated Caenorhabditis elegans aging analysis. Aging Cell 12:398-409. 